\title{
Clinicopathological Characteristics, Survival Outcomes, and Genetic Alterations of Younger Patient With Gastric Cancer: Results From China National Cancer Center and cBioPortal
}

\section{Penghui Niu}

Chinese Academy of Medical Sciences Cancer Institute and Hospital: Cancer Hospital Chinese Academy of Medical Sciences

Huang Huang

Chinese Academy of Medical Sciences Cancer Institute and Hospital: Cancer Hospital Chinese Academy of Medical Sciences Lulu Zhao

Chinese Academy of Medical Sciences Cancer Institute and Hospital: Cancer Hospital Chinese Academy of Medical Sciences

\section{Tongbo Wang}

Chinese Academy of Medical Sciences Cancer Institute and Hospital: Cancer Hospital Chinese Academy of Medical Sciences

\section{Xiaojie Zhang}

Chinese Academy of Medical Sciences Cancer Institute and Hospital: Cancer Hospital Chinese Academy of Medical Sciences Wanqing Wang

Chinese Academy of Medical Sciences Cancer Institute and Hospital: Cancer Hospital Chinese Academy of Medical Sciences Yawei Zhang

Chinese Academy of Medical Sciences Cancer Institute and Hospital: Cancer Hospital Chinese Academy of Medical Sciences Chunguang Guo

Chinese Academy of Medical Sciences Cancer Institute and Hospital: Cancer Hospital Chinese Academy of Medical Sciences Dongbing Zhao

Chinese Academy of Medical Sciences Cancer Institute and Hospital: Cancer Hospital Chinese Academy of Medical Sciences Ying-Tai Chen ( $\sim$ yingtaichen@126.com )

Chinese Academy of Medical Sciences Cancer Institute and Hospital: Cancer Hospital Chinese Academy of Medical Sciences https://orcid.org/0000-0002$4342-4335$

\section{Research Article}

Keywords: Gastric cancer, Younger patient, Clinicopathological characteristics, Survival outcomes, Genetic alterations

Posted Date: September 7th, 2021

DOl: https://doi.org/10.21203/rs.3.rs-862005/v1

License: @ (i) This work is licensed under a Creative Commons Attribution 4.0 International License. Read Full License

Version of Record: A version of this preprint was published at Cancer Medicine on March 22nd, 2022. See the published version at https://doi.org/10.1002/cam4.4669. 


\section{Abstract}

\section{Background}

Survival outcomes of younger patients with gastric cancer (GC) remains controversial. The present study sought to explore clinicopathological characteristics, survival outcomes, and genetic alterations in the younger and older patients with GC.

\section{Methods}

Patients with GC were identified from the China National Cancer Center Gastric Cancer Database (NCCGCDB) during 1998-2018. Survival analysis was conducted via Kaplan-Meier estimates and Cox proportional hazards models. Sequencing data were enrolled from the China National Cancer Center, TCGA, and MSKCC databases.

\section{Results}

A total of 1146 younger and 16988 older cases were included. Patients in the younger group were predominant in poor differentiation (53.7\% versus $33.8 \%$, $\mathrm{P}<0.0001)$, and pTNM stage IV (19.5\% versus $11.8 \%, \mathrm{P}<0.001)$. The 5 -year overall survival (OS) of patients in NCCGCDB has noticeable increased from 1998 to 2018. Younger age was an independent prognostic factor for GC patients in pTNM stage III $(P=0.014)$, while other stages showed no difference. Compared to the younger, older patients had a higher mutation frequency in $\angle R P 1 B, G N A S, A P C, K M T 2 D$ genes (all $\mathrm{P}<0.05$ ). In addition, although no significant difference, results of the China National Cancer Center, TCGA, and MSKCC cohorts indicated that younger patients dominated in CDH1, RHOA, and CTNNB1 mutations.

\section{Conclusions}

Stable proportion of younger cases and noticeable survival improvements were reported in the China National Cancer Center. Younger patients with pTNM stage III had a worse survival than older, while other tumor stages showed no difference. Furthermore, distinctive molecular characteristics were identified in younger GC patients, which might partly explain histopathological behaviors and prognosis of in this subpopulation.

\section{Background}

Gastric cancer (GC) remained one of the most frequent digestive cancers and represented the fourth leading cause of cancer-related death worldwide [1]. More than $40 \%$ of GC cases and deaths occurred in China, with an age-standardized 5-year survival rate of 35.1\% [2, 3, 4]. It was generally considered that GC mostly onset in patients aged 50-70 years, and younger patients only account for 2.0-6.2\% [5, 6]. Although the incidence of GC revealed a decline in the general population, concern has been raised about the stable or increased trends of younger patients in the last decades [7, 8, 9].

Younger patients with GC were more likely to exhibit distinctive biological behaviors, such as female predominance, poor differentiation, and advanced stage [10-13]. To date, results for the survival outcomes in younger patients with GC have been inconsistent [10-15]. Some studies reported that younger patients own a longer survival time than older $[10,11]$, whereas others showed no difference $[12,13]$. Several studies even revealed poor survival of GC in younger adults $[14,15]$. From the molecular perspective, the increased incidence of younger patients might owe to the distinctive pathogenesis, and certain genetic alterations have been demonstrated to play a critical role in the progression and prognosis of GC among age groups [16, 17]. However, recent knowledge regarding the mutation profiles in younger GC patients was limited, and a comprehensive molecular profile target younger patients should be defined to enhance understanding of molecular pathology in this subpopulation.

In this study, we sought to explore trends of clinicopathological characteristics and survival outcomes in younger and older patients from the China National Cancer Center over the past 20 years, which might serve as a reference for a large population-based study in China. Moreover, genetic alterations of younger patients were comprehensively investigated based on the China National Cancer Center and the cBioPortal for Cancer Genomics database, thus providing novel insights for the genomics and precision medicine of this subpopulation.

\section{Methods}

\section{Data source and patient selection}

The study population was described in detail in the previous research [18, 19]. Briefly, all histologically confirmed incident cases of GC diagnosed between 1998 and 2018 in the cancer center were identified through the China National Cancer Center Gastric Cancer Database (NCCGCDB). Enrollment criteria included age between 18 and 85 years, residence in China, and without a previous cancer diagnosis, except for GC. All GC cases that met the inclusion criteria were divided into two age groups: the younger group ( $<40$ years of age) and older group ( $\geq 40$ years of age). The rationale for younger group was to remain consistent with the majority of prior studies [11,20,21]. Clinical data abstracted from the NCCGCDB included patients' demographics, clinicopathological characteristics, and survival variables. Stage of GC was assessed according to the 8th edition of the American Joint Committee on Cancer (AJCC) TNM staging system. The primary endpoints of the study were OS and progression-free survival (PFS). According to the time of histological diagnosis, changing trends of the younger and older patients with GC were investigated in four consecutive periods: from 1998 to 2003 (period 1), from 2003 to 2008 (period 2), from 2008 to 2013 (period 3), and from 2013 to 2018 (period 4).

Of 29 patients with GC included in the further molecular analysis had their tumor specimens sequenced at our institution. All tumor samples were evaluated by pathologists prior to DNA extraction for sequencing. Moreover, we searched the online cBioPortal for Cancer Genomics (http://www.cbioportal.org/) to obtain high-volume genetic resources [19-22]. Finally, 8 gastric adenocarcinoma datasets from TCGA and MSKCC databases met our inclusion criteria, and a total of 1098 GC cases with clear clinical data and mutation detection by next-generation sequencing (NGS) were included in the comparative analysis. 


\section{DNA extraction and sequencing}

DNA from tumor samples and peripheral blood was extracted via the QIAamp DNA Mini Kit (QIAGEN, Valencia, CA) according to the manufacturer's instructions. QubitdsDNA assay was performed to measure the DNA concentration. A minimum of 50 ng of DNA is required for construction of an NGS library. For GC patients with available DNA, targeted DNA sequencing was performed. DNA was profiled by using a capture-based targeted sequencing panel (Burning Rock Biotech, Guangzhou, People's Republic of China), targeting 520 genes and spanning 1.67M of Human genomic regions.

\section{Sequencing data analysis}

Sequencing data were mapped to the human genome (hg19) using BWA aligner 0.7.10. Local alignment optimization and variant calling were performed using GATK v3.2-2, while DNA translocation analysis was performed using both Tophat2 and Factera 1.4.3. Different mutation calling thresholds were applied on samples with different DNA qualities to avoid false-positive mutation calls due to DNA damage. Single nucleotide variants (SNVs) and insertion-deletions (INDELs) indels identified were annotated using the dbNSFP(v30a), COSMIC (v69), and dbSNP (snp138) database. Variants with a global minor allele frequency greater than $1.0 \%$ in 1000 Genome Project (Phase3, http://www.1000genomes. org/data) were considered as common SNPs and removed. Tumor mutational burden (TMB) was defined as the number of somatic, coding, base substation, and indels per megabase of genome examined. Synonymous mutations were counted to reduce sampling noise. White blood cells were used to filter germline mutations.

\section{Statistical Analysis}

The bar chart was plotted to evaluate the variation in tumor stage for younger and older patients from 1998 to 2018 . The Chi-squared test was performed to compare categorical variables between the two groups, while continuous variables were evaluated using Student's t-test. OS and PFS for the younger and older group were calculated with the Kaplan-Meier method, while the log-rank test estimated the relevant survival discrepancy. Associations between risk factors and OS were investigated by univariate and multivariate Cox proportional hazard regression analysis, while the corresponding hazard ratio (HR) and $95 \% \mathrm{Cl}$ were generated. The covariates included in the final models were determined by using stepwise selection with minimized AIC. The significant level for adding variables was 0.05 , and the significance level for removing variables was 0.10 . Statistical significance was set at two-sided $P$ values less than 0.05 , and all analyses in the study were conducted using SAS software v9.4 (SAS Institute, Inc., Cary, NC).

\section{Results}

\section{Demographic and Clinicopathological features}

From 1998 to 2018 , a total of 1146 younger cases (6.3\%) and 16988 older cases (93.7\%) were finally included. Among all GC patients, 2035 were diagnosed in period 1, 3859 in period 2, 6054 in period 3, and 6150 in period 4 (As shown in Table 1). There were significant differences in the distribution of gender, smoking, alcohol history, BMI, primary tumor location, differentiation, pTNM stage between the younger group and older group (all P< 0.01 ). Distinctive demographic disparities among age groups were founded in the China National Cancer Center. Younger patients were predominant in females (50.1\% versus $21.6 \%, \mathrm{P}<0.0001)$. Conversely, relatively higher percentages of smokers $(21.1 \%$ versus $42.4 \%, P<0.0001)$, alcohol drinkers $(20.9 \%$ versus $34.5 \%, P<0.0001)$, and overweight/obesity $(\mathrm{BMI} \geq 23)(36.1 \%$ versus $53.4 \%, \mathrm{P}<0.0001)$ were revealed in older. 
Table 1

Demographic and clinicopathological characteristics between younger group and older group in 4 consecutive periods (bidirectional cohort 1998-2018).

\begin{tabular}{|c|c|c|c|c|c|c|c|c|c|c|c|c|c|}
\hline & \multicolumn{4}{|c|}{ Period 1 (1998-2003) } & \multicolumn{3}{|c|}{ Period 2 (2004-2008) } & \multicolumn{3}{|c|}{ Period 3 (2009-2013) } & \multicolumn{3}{|c|}{ Period 4 (2014-2018) } \\
\hline & \multicolumn{2}{|c|}{ Younger group } & \multirow{2}{*}{$\begin{array}{l}\text { Older } \\
\text { group } \\
\text { n (\%) }\end{array}$} & \multirow[t]{2}{*}{$P$ Value } & \multirow{2}{*}{$\begin{array}{l}\begin{array}{l}\text { Younger } \\
\text { group }\end{array} \\
\mathbf{n ( \% )}\end{array}$} & \multirow{2}{*}{$\begin{array}{l}\text { Older } \\
\text { group } \\
\mathrm{n}(\%)\end{array}$} & \multirow[t]{2}{*}{$P$ Value } & \multirow{2}{*}{$\begin{array}{l}\begin{array}{l}\text { Younger } \\
\text { group }\end{array} \\
\mathrm{n}(\%)\end{array}$} & \multirow{2}{*}{$\begin{array}{l}\text { Older } \\
\text { group } \\
\mathrm{n}(\%)\end{array}$} & \multirow[t]{2}{*}{$P$ Value } & \multirow{2}{*}{$\begin{array}{l}\begin{array}{l}\text { Younger } \\
\text { group }\end{array} \\
\mathrm{n}(\%)\end{array}$} & \multirow{2}{*}{$\begin{array}{l}\text { Older } \\
\text { group }\end{array}$} & \multirow[t]{2}{*}{$P I$} \\
\hline & n (\%) & & & & & & & & & & & & \\
\hline \multicolumn{14}{|l|}{ Gender } \\
\hline Male & & $\begin{array}{l}83 \\
(50.0 \%)\end{array}$ & $\begin{array}{l}1,367 \\
(73.14 \%)\end{array}$ & & $\begin{array}{l}112 \\
(46.1 \%)\end{array}$ & $\begin{array}{l}2,744 \\
(75.1 \%)\end{array}$ & & $\begin{array}{l}209 \\
(52.6 \%)\end{array}$ & $\begin{array}{l}4,321 \\
(76.4 \%)\end{array}$ & & $\begin{array}{l}168 \\
(49.4 \%)\end{array}$ & $\begin{array}{l}4,384 \\
(75.5 \%)\end{array}$ & \\
\hline Female & & $\begin{array}{l}83 \\
(50.0 \%)\end{array}$ & $\begin{array}{l}502 \\
(26.86 \%)\end{array}$ & $<0.0001$ & $\begin{array}{l}131 \\
(53.9 \%)\end{array}$ & $\begin{array}{l}908 \\
(24.9 \%)\end{array}$ & $<0.0001$ & $\begin{array}{l}188 \\
(47.4 \%)\end{array}$ & $\begin{array}{l}1,336 \\
(23.6 \%)\end{array}$ & $<0.0001$ & $\begin{array}{l}172 \\
(50.6 \%)\end{array}$ & $\begin{array}{l}1,426 \\
(24.5 \%)\end{array}$ & $<0$ \\
\hline \multicolumn{14}{|c|}{ Smoking history } \\
\hline No & & $\begin{array}{l}132 \\
(79.5 \%)\end{array}$ & $\begin{array}{l}1,180 \\
(63.1 \%)\end{array}$ & & $\begin{array}{l}195 \\
(80.3 \%)\end{array}$ & $\begin{array}{l}2,242 \\
(61.4 \%)\end{array}$ & & $\begin{array}{l}302 \\
(76.1 \%)\end{array}$ & $\begin{array}{l}3,110 \\
(55.0 \%)\end{array}$ & & $\begin{array}{l}241 \\
(70.9 \%)\end{array}$ & $\begin{array}{l}2,813 \\
(48.4 \%)\end{array}$ & \\
\hline Yes & & $\begin{array}{l}33 \\
(19.9 \%)\end{array}$ & $\begin{array}{l}670 \\
(35.9 \%)\end{array}$ & & $\begin{array}{l}39 \\
(16.1 \%)\end{array}$ & $\begin{array}{l}1,247 \\
(34.2 \%)\end{array}$ & & $\begin{array}{l}84 \\
(21.2 \%)\end{array}$ & $\begin{array}{l}2,393 \\
(42.3 \%)\end{array}$ & & $\begin{array}{l}86 \\
(25.3 \%)\end{array}$ & $\begin{array}{l}2,896 \\
(49.9 \%)\end{array}$ & \\
\hline Unknow & & $1(0.6 \%)$ & $\begin{array}{l}19 \\
(1.0 \%)\end{array}$ & 0.0001 & $9(3.7 \%)$ & $\begin{array}{l}163 \\
(4.5 \%)\end{array}$ & $<0.0001$ & $\begin{array}{l}11 \\
(2.8 \%)\end{array}$ & $\begin{array}{l}154 \\
(2.7 \%)\end{array}$ & $<0.0001$ & $\begin{array}{l}13 \\
(3.8 \%)\end{array}$ & $\begin{array}{l}101 \\
(1.7 \%)\end{array}$ & $<0$ \\
\hline
\end{tabular}

Drinking history

\begin{tabular}{|c|c|c|c|c|c|c|c|c|c|c|c|}
\hline No & $\begin{array}{l}131 \\
(78.9 \%)\end{array}$ & $\begin{array}{l}1,328 \\
(71.1 \%)\end{array}$ & & $\begin{array}{l}206 \\
(84.8 \%)\end{array}$ & $\begin{array}{l}2,562 \\
(70.2 \%)\end{array}$ & & $\begin{array}{l}304 \\
(76.6 \%)\end{array}$ & $\begin{array}{l}3,594 \\
(63.5 \%)\end{array}$ & & $\begin{array}{l}230 \\
(67.7 \%)\end{array}$ & $\begin{array}{l}3,190 \\
(54.9 \%)\end{array}$ \\
\hline Yes & $\begin{array}{l}34 \\
(20.5 \%)\end{array}$ & $\begin{array}{l}520 \\
(27.8 \%)\end{array}$ & & $\begin{array}{l}27 \\
(11.1 \%)\end{array}$ & $\begin{array}{l}919 \\
(25.2 \%)\end{array}$ & & $\begin{array}{l}81 \\
(20.4 \%)\end{array}$ & $\begin{array}{l}1,908 \\
(33.7 \%)\end{array}$ & & $\begin{array}{l}97 \\
(28.5 \%)\end{array}$ & $\begin{array}{l}2,519 \\
(43.4 \%)\end{array}$ \\
\hline Unknown & $1(0.6 \%)$ & $\begin{array}{l}21 \\
(1.1 \%)\end{array}$ & 0.095 & $\begin{array}{l}10 \\
(4.1 \%)\end{array}$ & $\begin{array}{l}171 \\
(4.7 \%)\end{array}$ & $<0.0001$ & $\begin{array}{l}12 \\
(3.0 \%)\end{array}$ & $\begin{array}{l}155 \\
(2.7 \%)\end{array}$ & $<0.0001$ & $\begin{array}{l}13 \\
(3.8 \%)\end{array}$ & $\begin{array}{l}101 \\
(1.7 \%)\end{array}$ \\
\hline
\end{tabular}

BMI $\left(\mathrm{kg} / \mathrm{m}^{2}\right)$ at

diagnosis

\begin{tabular}{|c|c|c|c|c|c|c|c|c|c|c|c|}
\hline$<18.5$ & $\begin{array}{l}29 \\
(17.5 \%)\end{array}$ & $\begin{array}{l}124 \\
(6.6 \%)\end{array}$ & & $\begin{array}{l}50 \\
(20.6 \%)\end{array}$ & $\begin{array}{l}264 \\
(7.2 \%)\end{array}$ & & $\begin{array}{l}52 \\
(13.1 \%)\end{array}$ & $\begin{array}{l}336 \\
(5.9 \%)\end{array}$ & & $\begin{array}{l}54 \\
(15.9 \%)\end{array}$ & $\begin{array}{l}283 \\
(4.9 \%)\end{array}$ \\
\hline $18.5-22.9$ & $\begin{array}{l}73 \\
(44.0 \%)\end{array}$ & $\begin{array}{l}733 \\
(39.2 \%)\end{array}$ & & $\begin{array}{l}109 \\
(44.9 \%)\end{array}$ & $\begin{array}{l}1,346 \\
(36.9 \%)\end{array}$ & & $\begin{array}{l}182 \\
(45.8 \%)\end{array}$ & $\begin{array}{l}2,162 \\
(38.2 \%)\end{array}$ & & $\begin{array}{l}143 \\
(42.1 \%)\end{array}$ & $\begin{array}{l}2,101 \\
(36.2 \%)\end{array}$ \\
\hline $23-27.4$ & $\begin{array}{l}48 \\
(28.9 \%)\end{array}$ & $\begin{array}{l}755 \\
(40.4 \%)\end{array}$ & & $\begin{array}{l}53 \\
(21.8 \%)\end{array}$ & $\begin{array}{l}1,388 \\
(38.0 \%)\end{array}$ & & $\begin{array}{l}110 \\
(27.7 \%)\end{array}$ & $\begin{array}{l}2,262 \\
(40.0 \%)\end{array}$ & & $\begin{array}{l}98 \\
(28.8 \%)\end{array}$ & $\begin{array}{l}2,574 \\
(44.3 \%)\end{array}$ \\
\hline$\geq 27.5$ & $9(5.4 \%)$ & $\begin{array}{l}196 \\
(10.5 \%)\end{array}$ & & $\begin{array}{l}17 \\
(7.0 \%)\end{array}$ & $\begin{array}{l}423 \\
(11.6 \%)\end{array}$ & & $\begin{array}{l}39 \\
(9.8 \%)\end{array}$ & $\begin{array}{l}701 \\
(12.4 \%)\end{array}$ & & $\begin{array}{l}40 \\
(11.8 \%)\end{array}$ & $\begin{array}{l}766 \\
(13.2 \%)\end{array}$ \\
\hline Unknown & $7(4.2 \%)$ & $\begin{array}{l}61 \\
(3.3 \%)\end{array}$ & $<0.0001$ & $\begin{array}{l}14 \\
(5.8 \%)\end{array}$ & $\begin{array}{l}231 \\
(6.3 \%)\end{array}$ & $<0.0001$ & $\begin{array}{l}14 \\
(3.5 \%)\end{array}$ & $\begin{array}{l}196 \\
(3.5 \%)\end{array}$ & $<0.0001$ & $5(1.5 \%)$ & $\begin{array}{l}86 \\
(1.5 \%)\end{array}$ \\
\hline
\end{tabular}

Weight loss

\begin{tabular}{|c|c|c|c|c|c|c|c|c|c|c|c|}
\hline None & $\begin{array}{l}61 \\
(36.8 \%)\end{array}$ & $\begin{array}{l}615 \\
(32.9 \%)\end{array}$ & & $\begin{array}{l}104 \\
(42.8 \%)\end{array}$ & $\begin{array}{l}1,427 \\
(39.1 \%)\end{array}$ & & $\begin{array}{l}241 \\
(60.7 \%)\end{array}$ & $\begin{array}{l}3,238 \\
(57.2 \%)\end{array}$ & & $\begin{array}{l}209 \\
(61.5 \%)\end{array}$ & $\begin{array}{l}3,504 \\
(60.3 \%)\end{array}$ \\
\hline$<10 \%$ & $\begin{array}{l}44 \\
(26.5 \%)\end{array}$ & $\begin{array}{l}461 \\
(24.7 \%)\end{array}$ & & $\begin{array}{l}50 \\
(20.6 \%)\end{array}$ & $\begin{array}{l}842 \\
(23.1 \%)\end{array}$ & & $\begin{array}{l}78 \\
(19.7 \%)\end{array}$ & $\begin{array}{l}1,419 \\
(25.1 \%)\end{array}$ & & $\begin{array}{l}74 \\
(21.8 \%)\end{array}$ & $\begin{array}{l}1,531 \\
(26.4 \%)\end{array}$ \\
\hline$\geq 10 \%$ & $\begin{array}{l}25 \\
(15.1 \%)\end{array}$ & $\begin{array}{l}191 \\
(10.2 \%)\end{array}$ & & $\begin{array}{l}23 \\
(9.5 \%)\end{array}$ & $\begin{array}{l}339 \\
(9.3 \%)\end{array}$ & & $\begin{array}{l}46 \\
(11.6 \%)\end{array}$ & $\begin{array}{l}547 \\
(9.7 \%)\end{array}$ & & $\begin{array}{l}37 \\
(10.9 \%)\end{array}$ & $\begin{array}{l}557 \\
(9.6 \%)\end{array}$ \\
\hline Unknown & $\begin{array}{l}36 \\
(22.7 \%)\end{array}$ & $\begin{array}{l}602 \\
(32.2 \%)\end{array}$ & 0.022 & $\begin{array}{l}66 \\
(27.2 \%)\end{array}$ & $\begin{array}{l}1,044 \\
(28.6 \%)\end{array}$ & 0.66 & $\begin{array}{l}32 \\
(8.1 \%)\end{array}$ & $\begin{array}{l}453 \\
(8.0 \%)\end{array}$ & 0.086 & $\begin{array}{l}20 \\
(5.9 \%)\end{array}$ & $\begin{array}{l}218 \\
(3.8 \%)\end{array}$ \\
\hline
\end{tabular}

H. pylori infection

\begin{tabular}{|c|c|c|c|c|c|c|c|c|c|c|c|}
\hline $\mathrm{Ne}$ & $0(0.0 \%)$ & \multicolumn{2}{|l|}{$3(0.2 \%)$} & $1(0.4 \%)$ & \multicolumn{2}{|l|}{$\begin{array}{l}51 \\
(1.4 \%)\end{array}$} & $\begin{array}{l}23 \\
(5.8 \%)\end{array}$ & \multicolumn{2}{|l|}{$\begin{array}{l}397 \\
(7.0 \%)\end{array}$} & $\begin{array}{l}59 \\
(17.4 \%)\end{array}$ & $\begin{array}{l}1,004 \\
(17.3 \%)\end{array}$ \\
\hline Positive & 0 (0.0\%) & $\begin{array}{l}11 \\
(0.6 \%)\end{array}$ & & $2(0.8 \%)$ & $\begin{array}{l}42 \\
(1.2 \%)\end{array}$ & & $\begin{array}{l}38 \\
(9.6 \%)\end{array}$ & $\begin{array}{l}522 \\
(9.2 \%)\end{array}$ & & $\begin{array}{l}27 \\
(7.9 \%)\end{array}$ & $\begin{array}{l}524 \\
(9.0 \%)\end{array}$ \\
\hline Unknown & $\begin{array}{l}166 \\
(100.0 \%)\end{array}$ & $\begin{array}{l}1,855 \\
(99.3 \%)\end{array}$ & 1.00 & $\begin{array}{l}240 \\
(98.8 \%)\end{array}$ & $\begin{array}{l}3,559 \\
(97.5 \%)\end{array}$ & 0.50 & $\begin{array}{l}336 \\
(84.6 \%)\end{array}$ & $\begin{array}{l}4,738 \\
(83.8 \%)\end{array}$ & 0.64 & $\begin{array}{l}254 \\
(74.7 \%)\end{array}$ & $\begin{array}{l}4,282 \\
(73.7 \%)\end{array}$ \\
\hline
\end{tabular}

Primary tumor

location

Proximal $\begin{array}{ll}32 & 1,403 \\ (13.2 \%) & (38.4 \%)\end{array}$

Page $4 / 19$ 


\begin{tabular}{|c|c|c|c|c|c|c|c|c|c|c|c|c|}
\hline Distal & $\begin{array}{l}126 \\
(75.9 \%)\end{array}$ & $\begin{array}{l}898 \\
(48.1 \%)\end{array}$ & & $\begin{array}{l}198 \\
(81.5 \%)\end{array}$ & $\begin{array}{l}1,996 \\
(54.7 \%)\end{array}$ & & $\begin{array}{l}296 \\
(74.6 \%)\end{array}$ & $\begin{array}{l}3,082 \\
(54.5 \%)\end{array}$ & & $\begin{array}{l}265 \\
(77.9 \%)\end{array}$ & $\begin{array}{l}3,105 \\
(53.4 \%)\end{array}$ & \\
\hline $\begin{array}{l}\text { Overlapping } \\
\text { lesions }\end{array}$ & $6(3.6 \%)$ & $\begin{array}{l}85 \\
(4.6 \%)\end{array}$ & & $3(1.2 \%)$ & $\begin{array}{l}94 \\
(2.6 \%)\end{array}$ & & $\begin{array}{l}19 \\
(4.8 \%)\end{array}$ & $\begin{array}{l}250 \\
(4.4 \%)\end{array}$ & & $\begin{array}{l}15 \\
(4.4 \%)\end{array}$ & $\begin{array}{l}422 \\
(7.3 \%)\end{array}$ & \\
\hline Unknown & $6(3.6 \%)$ & $\begin{array}{l}50 \\
(2.7 \%)\end{array}$ & $<0.0001$ & $\begin{array}{l}10 \\
(4.1 \%)\end{array}$ & $\begin{array}{l}159 \\
(4.4 \%)\end{array}$ & $<0.0001$ & $\begin{array}{l}17 \\
(4.3 \%)\end{array}$ & $\begin{array}{l}161 \\
(2.9 \%)\end{array}$ & $<0.0001$ & $\begin{array}{l}11 \\
(3.2 \%)\end{array}$ & $\begin{array}{l}168 \\
(2.9 \%)\end{array}$ & $<0$. \\
\hline \multicolumn{13}{|l|}{ Lauren classification } \\
\hline Intestinal & $0(0.0 \%)$ & $0(0.0 \%)$ & & $0(0.0 \%)$ & $\begin{array}{l}1 \\
(0.0 \%)\end{array}$ & & $\begin{array}{l}16 \\
(4.0 \%)\end{array}$ & $\begin{array}{l}1,073 \\
(19.0 \%)\end{array}$ & & $\begin{array}{l}23 \\
(6.8 \%)\end{array}$ & $\begin{array}{l}1,724 \\
(29.7 \%)\end{array}$ & \\
\hline Diffuse & $0(0.0 \%)$ & $1(0.1 \%)$ & & $0(0.0 \%)$ & $\begin{array}{l}1 \\
(0.0 \%)\end{array}$ & & $\begin{array}{l}111 \\
(28.0 \%)\end{array}$ & $\begin{array}{l}942 \\
(16.7 \%)\end{array}$ & & $\begin{array}{l}173 \\
(50.9 \%)\end{array}$ & $\begin{array}{l}1,380 \\
(23.8 \%)\end{array}$ & \\
\hline Mixed & $0(0.0 \%)$ & $1(0.1 \%)$ & & $0(0.0 \%)$ & $\begin{array}{l}4 \\
(0.1 \%)\end{array}$ & & $\begin{array}{l}25 \\
(6.3 \%)\end{array}$ & $\begin{array}{l}607 \\
(10.7 \%)\end{array}$ & & $\begin{array}{l}47 \\
(13.8 \%)\end{array}$ & $\begin{array}{l}1,115 \\
(19.2 \%)\end{array}$ & \\
\hline Unknown & $\begin{array}{l}166 \\
(100.0 \%)\end{array}$ & $\begin{array}{l}1,867 \\
(99.9 \%)\end{array}$ & 1.00 & $\begin{array}{l}243 \\
(100.0 \%)\end{array}$ & $\begin{array}{l}3,646 \\
(99.8 \%)\end{array}$ & 1.00 & $\begin{array}{l}245 \\
(61.7 \%)\end{array}$ & $\begin{array}{l}3,035 \\
(53.7 \%)\end{array}$ & $<0.0001$ & $\begin{array}{l}97 \\
(28.5 \%)\end{array}$ & $\begin{array}{l}1,591 \\
(27.4 \%)\end{array}$ & $<0$. \\
\hline \multicolumn{13}{|l|}{$\begin{array}{l}\text { Borrmann } \\
\text { classification }\end{array}$} \\
\hline Borrmann I & $\begin{array}{l}13 \\
(7.8 \%)\end{array}$ & $\begin{array}{l}210 \\
(11.2 \%)\end{array}$ & & $6(2.5 \%)$ & $\begin{array}{l}316 \\
(8.7 \%)\end{array}$ & & $\begin{array}{l}30 \\
(7.6 \%)\end{array}$ & $\begin{array}{l}537 \\
(9.5 \%)\end{array}$ & & $\begin{array}{l}29 \\
(8.5 \%)\end{array}$ & $\begin{array}{l}587 \\
(10.1 \%)\end{array}$ & \\
\hline Borrmann II & $\begin{array}{l}45 \\
(27.1 \%)\end{array}$ & $\begin{array}{l}750 \\
(40.1 \%)\end{array}$ & & $\begin{array}{l}96 \\
(39.5 \%)\end{array}$ & $\begin{array}{l}1,327 \\
(36.3 \%)\end{array}$ & & $\begin{array}{l}134 \\
(33.8 \%)\end{array}$ & $\begin{array}{l}2,115 \\
(37.4 \%)\end{array}$ & & $\begin{array}{l}107 \\
(31.5 \%)\end{array}$ & $\begin{array}{l}2,085 \\
(35.9 \%)\end{array}$ & \\
\hline Borrmann III & $\begin{array}{l}52 \\
(31.3 \%)\end{array}$ & $\begin{array}{l}400 \\
(21.4 \%)\end{array}$ & & $\begin{array}{l}52 \\
(21.4 \%)\end{array}$ & $\begin{array}{l}859 \\
(23.5 \%)\end{array}$ & & $\begin{array}{l}87 \\
(21.9 \%)\end{array}$ & $\begin{array}{l}1,304 \\
(23.1 \%)\end{array}$ & & $\begin{array}{l}93 \\
(27.4 \%)\end{array}$ & $\begin{array}{l}1,666 \\
(28.7 \%)\end{array}$ & \\
\hline Borrmann IV & $\begin{array}{l}16 \\
(9.6 \%)\end{array}$ & $\begin{array}{l}116 \\
(6.2 \%)\end{array}$ & & $\begin{array}{l}23 \\
(9.5 \%)\end{array}$ & $\begin{array}{l}306 \\
(8.4 \%)\end{array}$ & & $\begin{array}{l}37 \\
(9.3 \%)\end{array}$ & $\begin{array}{l}316 \\
(5.6 \%)\end{array}$ & & $\begin{array}{l}20 \\
(5.9 \%)\end{array}$ & $\begin{array}{l}250 \\
(4.3 \%)\end{array}$ & \\
\hline Mixed & $2(1.2 \%)$ & $7(0.4 \%)$ & & $0(0.0 \%)$ & $\begin{array}{l}9 \\
(0.3 \%)\end{array}$ & & $1(0.3 \%)$ & $\begin{array}{l}12 \\
(0.2 \%)\end{array}$ & & $\begin{array}{l}11 \\
(3.2 \%)\end{array}$ & $\begin{array}{l}150 \\
(2.6 \%)\end{array}$ & \\
\hline Unknown & $\begin{array}{l}38 \\
(22.9 \%)\end{array}$ & $\begin{array}{l}386 \\
(20.7 \%)\end{array}$ & 0.0010 & $\begin{array}{l}66 \\
(27.2 \%)\end{array}$ & $\begin{array}{l}835 \\
(22.9 \%)\end{array}$ & 0.014 & $\begin{array}{l}108 \\
(27.2 \%)\end{array}$ & $\begin{array}{l}1,373 \\
(24.3 \%)\end{array}$ & 0.022 & $\begin{array}{l}80 \\
(23.5 \%)\end{array}$ & $\begin{array}{l}1,072 \\
(18.5 \%)\end{array}$ & 0.0 \\
\hline \multicolumn{13}{|l|}{ Linitis plastica } \\
\hline No & $\begin{array}{l}162 \\
(97.6 \%)\end{array}$ & $\begin{array}{l}1,851 \\
(99.0 \%)\end{array}$ & & $\begin{array}{l}232 \\
(95.5 \%)\end{array}$ & $\begin{array}{l}3,474 \\
(95.1 \%)\end{array}$ & & $\begin{array}{l}359 \\
(90.4 \%)\end{array}$ & $\begin{array}{l}5,312 \\
(93.9 \%)\end{array}$ & & $\begin{array}{l}321 \\
(94.4 \%)\end{array}$ & $\begin{array}{l}5,565 \\
(95.8 \%)\end{array}$ & \\
\hline Yes & $4(2.4 \%)$ & $9(0.5 \%)$ & & $1(0.4 \%)$ & $\begin{array}{l}13 \\
(0.4 \%)\end{array}$ & & $\begin{array}{l}13 \\
(3.3 \%)\end{array}$ & $\begin{array}{l}57 \\
(1.0 \%)\end{array}$ & & $\begin{array}{l}11 \\
(3.2 \%)\end{array}$ & $\begin{array}{l}72 \\
(1.2 \%)\end{array}$ & \\
\hline Unknown & $0(0.0 \%)$ & $9(0.5 \%)$ & 0.028 & $\begin{array}{l}10 \\
(4.1 \%)\end{array}$ & $\begin{array}{l}165 \\
(4.5 \%)\end{array}$ & 0.95 & $\begin{array}{l}25 \\
(6.3 \%)\end{array}$ & $\begin{array}{l}288 \\
(5.1 \%)\end{array}$ & 0.0001 & $8(2.4 \%)$ & $\begin{array}{l}173 \\
(3.0 \%)\end{array}$ & 0.0 \\
\hline \multicolumn{13}{|l|}{ Differentiation } \\
\hline Well & $1(0.6 \%)$ & $\begin{array}{l}44 \\
(2.4 \%)\end{array}$ & & $0(0.0 \%)$ & $\begin{array}{l}60 \\
(1.6 \%)\end{array}$ & & $4(1.0 \%)$ & $\begin{array}{l}126 \\
(2.2 \%)\end{array}$ & & $1(0.3 \%)$ & $\begin{array}{l}130 \\
(2.2 \%)\end{array}$ & \\
\hline Moderate & $\begin{array}{l}21 \\
(12.7 \%)\end{array}$ & $\begin{array}{l}658 \\
(35.2 \%)\end{array}$ & & $\begin{array}{l}26 \\
(10.7 \%)\end{array}$ & $\begin{array}{l}1,162 \\
(31.8 \%)\end{array}$ & & $\begin{array}{l}34 \\
(8.6 \%)\end{array}$ & $\begin{array}{l}1,882 \\
(33.3 \%)\end{array}$ & & $\begin{array}{l}42 \\
(12.4 \%)\end{array}$ & $\begin{array}{l}2,167 \\
(37.3 \%)\end{array}$ & \\
\hline Poor & $\begin{array}{l}87 \\
(52.4 \%)\end{array}$ & $\begin{array}{l}575 \\
(30.8 \%)\end{array}$ & & $\begin{array}{l}116 \\
(47.7 \%)\end{array}$ & $\begin{array}{l}1,179 \\
(32.3 \%)\end{array}$ & & $\begin{array}{l}216 \\
(54.4 \%)\end{array}$ & $\begin{array}{l}1,931 \\
(34.1 \%)\end{array}$ & & $\begin{array}{l}196 \\
(57.7 \%)\end{array}$ & $\begin{array}{l}2,054 \\
(35.4 \%)\end{array}$ & \\
\hline Undifferentiated & $0(0.0 \%)$ & $1(0.1 \%)$ & & $0(0.0 \%)$ & $\begin{array}{l}0 \\
(0.0 \%)\end{array}$ & & $0(0.0 \%)$ & $\begin{array}{l}2 \\
(0.0 \%)\end{array}$ & & $0(0.0 \%)$ & $\begin{array}{l}1 \\
(0.0 \%)\end{array}$ & \\
\hline Unknown & $\begin{array}{l}57 \\
(34.3 \%)\end{array}$ & $\begin{array}{l}591 \\
(31.6 \%)\end{array}$ & $<0.0001$ & $\begin{array}{l}101 \\
(41.6 \%)\end{array}$ & $\begin{array}{l}1,251 \\
(34.3 \%)\end{array}$ & $<0.0001$ & $\begin{array}{l}143 \\
(36.0 \%)\end{array}$ & $\begin{array}{l}1,716 \\
(30.3 \%)\end{array}$ & $<0.0001$ & $\begin{array}{l}101 \\
(29.7 \%)\end{array}$ & $\begin{array}{l}1,458 \\
(25.1 \%)\end{array}$ & $<0$. \\
\hline \multicolumn{13}{|l|}{ HER2 score } \\
\hline $0(-)$ & $1(0.6 \%)$ & $0(0.0 \%)$ & & $3(1.2 \%)$ & $\begin{array}{l}19 \\
(0.5 \%)\end{array}$ & & $\begin{array}{l}90 \\
(22.7 \%)\end{array}$ & $\begin{array}{l}1,231 \\
(21.8 \%)\end{array}$ & & $\begin{array}{l}120 \\
(35.3 \%)\end{array}$ & $\begin{array}{l}2,020 \\
(34.8 \%)\end{array}$ & \\
\hline $1(+)$ & $0(0.0 \%)$ & $1(0.1 \%)$ & & $0(0.0 \%)$ & $\begin{array}{l}3 \\
(0.1 \%)\end{array}$ & & $\begin{array}{l}96 \\
(24.2 \%)\end{array}$ & $\begin{array}{l}1,291 \\
(22.8 \%)\end{array}$ & & $\begin{array}{l}107 \\
(31.5 \%)\end{array}$ & $\begin{array}{l}1,523 \\
(26.2 \%)\end{array}$ & \\
\hline $2(++)$ & $0(0.0 \%)$ & $1(0.1 \%)$ & & $0(0.0 \%)$ & $\begin{array}{l}3 \\
(0.1 \%)\end{array}$ & & $\begin{array}{l}27 \\
(6.8 \%)\end{array}$ & $\begin{array}{l}531 \\
(9.4 \%)\end{array}$ & & $\begin{array}{l}19 \\
(5.6 \%)\end{array}$ & $\begin{array}{l}673 \\
(11.6 \%)\end{array}$ & \\
\hline $3(+++)$ & $0(0.0 \%)$ & $0(0.0 \%)$ & & $0(0.0 \%)$ & $\begin{array}{l}1 \\
(0.0 \%)\end{array}$ & & $\begin{array}{l}12 \\
(3.0 \%)\end{array}$ & $\begin{array}{l}293 \\
(5.2 \%)\end{array}$ & & $7(2.1 \%)$ & $\begin{array}{l}288 \\
(5.0 \%)\end{array}$ & \\
\hline Unknown & $\begin{array}{l}165 \\
(99.4 \%)\end{array}$ & $\begin{array}{l}1,867 \\
(99.9 \%)\end{array}$ & 0.23 & $\begin{array}{l}240 \\
(98.8 \%)\end{array}$ & $\begin{array}{l}3,626 \\
(99.3 \%)\end{array}$ & 0.46 & $\begin{array}{l}172 \\
(43.3 \%)\end{array}$ & $\begin{array}{l}2,311 \\
(40.9 \%)\end{array}$ & 0.13 & $\begin{array}{l}87 \\
(25.6 \%)\end{array}$ & $\begin{array}{l}1,306 \\
(22.5 \%)\end{array}$ & 0.0 \\
\hline \multicolumn{13}{|c|}{ Page 5/19 } \\
\hline
\end{tabular}


Pathologic T-stage

\begin{tabular}{|c|c|c|c|c|c|c|c|c|c|c|c|}
\hline $\mathrm{T} 0+\mathrm{Tis}$ & $0(0.0 \%)$ & $1(0.1 \%)$ & & $2(0.8 \%)$ & $\begin{array}{l}13 \\
(0.4 \%)\end{array}$ & & $1(0.3 \%)$ & $\begin{array}{l}20 \\
(0.4 \%)\end{array}$ & & $0(0.0 \%)$ & $\begin{array}{l}24 \\
(0.4 \%)\end{array}$ \\
\hline T1 & $\begin{array}{l}17 \\
(10.2 \%)\end{array}$ & $\begin{array}{l}159 \\
(8.5 \%)\end{array}$ & & $\begin{array}{l}37 \\
(15.2 \%)\end{array}$ & $\begin{array}{l}357 \\
(9.8 \%)\end{array}$ & & $\begin{array}{l}59 \\
(14.9 \%)\end{array}$ & $\begin{array}{l}796 \\
(14.1 \%)\end{array}$ & & $\begin{array}{l}81 \\
(23.8 \%)\end{array}$ & $\begin{array}{l}1,269 \\
(21.8 \%)\end{array}$ \\
\hline T2 & $\begin{array}{l}13 \\
(7.8 \%)\end{array}$ & $\begin{array}{l}135 \\
(7.2 \%)\end{array}$ & & $\begin{array}{l}15 \\
(6.2 \%)\end{array}$ & $\begin{array}{l}248 \\
(6.8 \%)\end{array}$ & & $\begin{array}{l}30 \\
(7.6 \%)\end{array}$ & $\begin{array}{l}491 \\
(8.7 \%)\end{array}$ & & $\begin{array}{l}36 \\
(10.6 \%)\end{array}$ & $\begin{array}{l}554 \\
(9.5 \%)\end{array}$ \\
\hline T3 & $2(1.2 \%)$ & $\begin{array}{l}13 \\
(0.7 \%)\end{array}$ & & $\begin{array}{l}22 \\
(9.1 \%)\end{array}$ & $\begin{array}{l}311 \\
(8.5 \%)\end{array}$ & & $\begin{array}{l}80 \\
(20.2 \%)\end{array}$ & $\begin{array}{l}1,712 \\
(30.3 \%)\end{array}$ & & $\begin{array}{l}39 \\
(11.5 \%)\end{array}$ & $\begin{array}{l}1,159 \\
(20.0 \%)\end{array}$ \\
\hline T4 & $\begin{array}{l}98 \\
(59.0 \%)\end{array}$ & $\begin{array}{l}1,156 \\
(61.9 \%)\end{array}$ & & $\begin{array}{l}110 \\
(45.3 \%)\end{array}$ & $\begin{array}{l}1,903 \\
(52.1 \%)\end{array}$ & & $\begin{array}{l}116 \\
(29.2 \%)\end{array}$ & $\begin{array}{l}1,374 \\
(24.3 \%)\end{array}$ & & $\begin{array}{l}114 \\
(33.5 \%)\end{array}$ & $\begin{array}{l}1,654 \\
(28.5 \%)\end{array}$ \\
\hline TX & $\begin{array}{l}36 \\
(21.7 \%)\end{array}$ & $\begin{array}{l}405 \\
(21.7 \%)\end{array}$ & 0.74 & $\begin{array}{l}57 \\
(23.5 \%)\end{array}$ & $\begin{array}{l}820 \\
(22.5 \%)\end{array}$ & 0.066 & $\begin{array}{l}111 \\
(28.0 \%)\end{array}$ & $\begin{array}{l}1,264 \\
(22.3 \%)\end{array}$ & 0.0004 & $\begin{array}{l}70 \\
(20.6 \%)\end{array}$ & $\begin{array}{l}1,150 \\
(19.8 \%)\end{array}$ \\
\hline
\end{tabular}

Pathologic N-stage

\begin{tabular}{|c|c|c|c|c|c|c|c|c|c|c|c|}
\hline NO & $\begin{array}{l}27 \\
(16.3 \%)\end{array}$ & $\begin{array}{l}418 \\
(22.4 \%)\end{array}$ & & $\begin{array}{l}62 \\
(25.5 \%)\end{array}$ & $\begin{array}{l}830 \\
(22.7 \%)\end{array}$ & & $\begin{array}{l}97 \\
(24.4 \%)\end{array}$ & $\begin{array}{l}1,518 \\
(26.8 \%)\end{array}$ & & $\begin{array}{l}110 \\
(32.4 \%)\end{array}$ & $\begin{array}{l}1,893 \\
(32.6 \%)\end{array}$ \\
\hline $\mathrm{N} 1$ & $\begin{array}{l}21 \\
(12.7 \%)\end{array}$ & $\begin{array}{l}273 \\
(14.6 \%)\end{array}$ & & $\begin{array}{l}31 \\
(12.8 \%)\end{array}$ & $\begin{array}{l}520 \\
(14.2 \%)\end{array}$ & & $\begin{array}{l}41 \\
(10.3 \%)\end{array}$ & $\begin{array}{l}775 \\
(13.7 \%)\end{array}$ & & $\begin{array}{l}31 \\
(9.1 \%)\end{array}$ & $\begin{array}{l}729 \\
(12.6 \%)\end{array}$ \\
\hline N2 & $\begin{array}{l}29 \\
(17.5 \%)\end{array}$ & $\begin{array}{l}310 \\
(16.6 \%)\end{array}$ & & $\begin{array}{l}24 \\
(9.9 \%)\end{array}$ & $\begin{array}{l}510 \\
(14.0 \%)\end{array}$ & & $\begin{array}{l}52 \\
(13.1 \%)\end{array}$ & $\begin{array}{l}797 \\
(14.1 \%)\end{array}$ & & $\begin{array}{l}55 \\
(16.2 \%)\end{array}$ & $\begin{array}{l}766 \\
(13.2 \%)\end{array}$ \\
\hline N3 & $\begin{array}{l}50 \\
(30.1 \%)\end{array}$ & $\begin{array}{l}459 \\
(24.6 \%)\end{array}$ & & $\begin{array}{l}64 \\
(25.3 \%)\end{array}$ & $\begin{array}{l}888 \\
(24.3 \%)\end{array}$ & & $\begin{array}{l}91 \\
(22.9 \%)\end{array}$ & $\begin{array}{l}1,222 \\
(21.6 \%)\end{array}$ & & $\begin{array}{l}70 \\
(20.6 \%)\end{array}$ & $\begin{array}{l}1,195 \\
(20.6 \%)\end{array}$ \\
\hline$N X$ & $\begin{array}{l}39 \\
(23.5 \%)\end{array}$ & $\begin{array}{l}409 \\
(21.9 \%)\end{array}$ & 0.27 & $\begin{array}{l}62 \\
(25.5 \%)\end{array}$ & $\begin{array}{l}904 \\
(24.8 \%)\end{array}$ & 0.36 & $\begin{array}{l}116 \\
(29.2 \%)\end{array}$ & $\begin{array}{l}1,345 \\
(23.8 \%)\end{array}$ & 0.060 & $\begin{array}{l}74 \\
(21.8 \%)\end{array}$ & $\begin{array}{l}1,227 \\
(21.1 \%)\end{array}$ \\
\hline
\end{tabular}

Pathologic M-stage

\begin{tabular}{|c|c|c|c|c|c|c|c|c|c|c|c|}
\hline MO & $\begin{array}{l}122 \\
(73.5 \%)\end{array}$ & $\begin{array}{l}1,563 \\
(83.6 \%)\end{array}$ & & $\begin{array}{l}178 \\
(73.3 \%)\end{array}$ & $\begin{array}{l}2,939 \\
(80.5 \%)\end{array}$ & & $\begin{array}{l}284 \\
(71.5 \%)\end{array}$ & $\begin{array}{l}4,517 \\
(79.9 \%)\end{array}$ & & $\begin{array}{l}266 \\
(78.2 \%)\end{array}$ & $\begin{array}{l}4,882 \\
(84.0 \%)\end{array}$ \\
\hline M1 & $\begin{array}{l}40 \\
(24.1 \%)\end{array}$ & $\begin{array}{l}261 \\
(14.0 \%)\end{array}$ & & $\begin{array}{l}48 \\
\text { (19.8\%) }\end{array}$ & $\begin{array}{l}430 \\
(11.8 \%)\end{array}$ & & $\begin{array}{l}81 \\
(20.4 \%)\end{array}$ & $\begin{array}{l}714 \\
(12.6 \%)\end{array}$ & & $\begin{array}{l}54 \\
(15.9 \%)\end{array}$ & $\begin{array}{l}601 \\
(10.3 \%)\end{array}$ \\
\hline $\mathrm{MX}$ & $4(2.4 \%)$ & $\begin{array}{l}45 \\
(2.4 \%)\end{array}$ & 0.0020 & $\begin{array}{l}17 \\
(7.0 \%)\end{array}$ & $\begin{array}{l}283 \\
(7.8 \%)\end{array}$ & 0.0012 & $\begin{array}{l}32 \\
(8.1 \%)\end{array}$ & $\begin{array}{l}426 \\
(7.5 \%)\end{array}$ & $<0.0001$ & $\begin{array}{l}20 \\
(5.9 \%)\end{array}$ & $\begin{array}{l}327 \\
(5.6 \%)\end{array}$ \\
\hline
\end{tabular}

pTNM stage

\begin{tabular}{|c|c|c|c|c|c|c|c|c|c|c|c|}
\hline 0 & $0(0.0 \%)$ & $1(0.1 \%)$ & & $2(0.8 \%)$ & $\begin{array}{l}12 \\
(0.3 \%)\end{array}$ & & $1(0.3 \%)$ & $\begin{array}{l}20 \\
(0.4 \%)\end{array}$ & & $0(0.0 \%)$ & $\begin{array}{l}19 \\
(0.3 \%)\end{array}$ \\
\hline I & $\begin{array}{l}17 \\
(10.2 \%)\end{array}$ & $\begin{array}{l}210 \\
(11.2 \%)\end{array}$ & & $\begin{array}{l}42 \\
(17.3 \%)\end{array}$ & $\begin{array}{l}470 \\
(12.9 \%)\end{array}$ & & $\begin{array}{l}71 \\
(17.9 \%)\end{array}$ & $\begin{array}{l}1,010 \\
(17.9 \%)\end{array}$ & & $\begin{array}{l}94 \\
(27.7 \%)\end{array}$ & $\begin{array}{l}1,422 \\
(24.5 \%)\end{array}$ \\
\hline ॥ & $\begin{array}{l}10 \\
(6.0 \%)\end{array}$ & $\begin{array}{l}63 \\
(3.4 \%)\end{array}$ & & $\begin{array}{l}24 \\
(9.9 \%)\end{array}$ & $\begin{array}{l}279 \\
(7.6 \%)\end{array}$ & & $\begin{array}{l}40 \\
(10.1 \%)\end{array}$ & $\begin{array}{l}948 \\
(16.8 \%)\end{array}$ & & $\begin{array}{l}34 \\
(10.0 \%)\end{array}$ & $\begin{array}{l}935 \\
(16.1 \%)\end{array}$ \\
\hline III & $\begin{array}{l}85 \\
(51.2 \%)\end{array}$ & $\begin{array}{l}1,103 \\
(59.0 \%)\end{array}$ & & $\begin{array}{l}99 \\
(40.7 \%)\end{array}$ & $\begin{array}{l}1,895 \\
(51.9 \%)\end{array}$ & & $\begin{array}{l}153 \\
(38.5 \%)\end{array}$ & $\begin{array}{l}2,182 \\
(38.6 \%)\end{array}$ & & $\begin{array}{l}128 \\
(37.7 \%)\end{array}$ & $\begin{array}{l}2,048 \\
(35.3 \%)\end{array}$ \\
\hline IV & $\begin{array}{l}40 \\
(24.1 \%)\end{array}$ & $\begin{array}{l}261 \\
(14.0 \%)\end{array}$ & & $\begin{array}{l}48 \\
(19.8 \%)\end{array}$ & $\begin{array}{l}430 \\
(11.8 \%)\end{array}$ & & $\begin{array}{l}81 \\
(20.4 \%)\end{array}$ & $\begin{array}{l}714 \\
(12.6 \%)\end{array}$ & & $\begin{array}{l}54 \\
(15.9 \%)\end{array}$ & $\begin{array}{l}601 \\
(10.3 \%)\end{array}$ \\
\hline Unknown & $\begin{array}{l}14 \\
(8.4 \%)\end{array}$ & $\begin{array}{l}231 \\
(12.4 \%)\end{array}$ & 0.0039 & $\begin{array}{l}28 \\
(11.5 \%)\end{array}$ & $\begin{array}{l}566 \\
(15.5 \%)\end{array}$ & $<0.0001$ & $\begin{array}{l}51 \\
(12.9 \%)\end{array}$ & $\begin{array}{l}783 \\
(13.8 \%)\end{array}$ & $<0.0001$ & $\begin{array}{l}30 \\
(8.8 \%)\end{array}$ & $\begin{array}{l}785 \\
(13.5 \%)\end{array}$ \\
\hline
\end{tabular}

Lymphatic invasion

\begin{tabular}{|c|c|c|c|c|c|c|c|c|c|c|c|}
\hline No & $\begin{array}{l}76 \\
(45.8 \%)\end{array}$ & $\begin{array}{l}986 \\
(52.8 \%)\end{array}$ & & $\begin{array}{l}123 \\
(50.6 \%)\end{array}$ & $\begin{array}{l}1,777 \\
(48.7 \%)\end{array}$ & & $\begin{array}{l}183 \\
(46.1 \%)\end{array}$ & $\begin{array}{l}2,830 \\
(50.0 \%)\end{array}$ & & $\begin{array}{l}152 \\
(44.7 \%)\end{array}$ & $\begin{array}{l}2,384 \\
(41.0 \%)\end{array}$ \\
\hline Yes & $\begin{array}{l}36 \\
(21.7 \%)\end{array}$ & $\begin{array}{l}357 \\
(19.1 \%)\end{array}$ & & $\begin{array}{l}34 \\
(14.0 \%)\end{array}$ & $\begin{array}{l}749 \\
(20.5 \%)\end{array}$ & & $\begin{array}{l}75 \\
(18.9 \%)\end{array}$ & $\begin{array}{l}1,182 \\
(20.9 \%)\end{array}$ & & $\begin{array}{l}82 \\
(24.1 \%)\end{array}$ & $\begin{array}{l}1,959 \\
(33.7 \%)\end{array}$ \\
\hline Unknown & $\begin{array}{l}54 \\
(32.5 \%)\end{array}$ & $\begin{array}{l}526 \\
(28.1 \%)\end{array}$ & 0.23 & $\begin{array}{l}86 \\
(35.4 \%)\end{array}$ & $\begin{array}{l}1,126 \\
(30.8 \%)\end{array}$ & 0.039 & $\begin{array}{l}139 \\
(35.0 \%)\end{array}$ & $\begin{array}{l}1,645 \\
(29.1 \%)\end{array}$ & 0.043 & $\begin{array}{l}106 \\
(31.2 \%)\end{array}$ & $\begin{array}{l}1,467 \\
(25.3 \%)\end{array}$ \\
\hline
\end{tabular}

Vascular invasion

\begin{tabular}{|c|c|c|c|c|c|c|c|c|}
\hline No & $\begin{array}{l}76 \\
(45.8 \%)\end{array}$ & $\begin{array}{l}988 \\
(52.9 \%)\end{array}$ & $\begin{array}{l}124 \\
(51.0 \%)\end{array}$ & $\begin{array}{l}1,776 \\
(48.6 \%)\end{array}$ & $\begin{array}{l}183 \\
(46.1 \%)\end{array}$ & $\begin{array}{l}2,828 \\
(50.0 \%)\end{array}$ & $\begin{array}{l}152 \\
(44.7 \%)\end{array}$ & $\begin{array}{l}2,375 \\
(40.9 \%)\end{array}$ \\
\hline
\end{tabular}




\begin{tabular}{|c|c|c|c|c|c|c|c|c|c|c|c|c|}
\hline Unknown & $\begin{array}{l}54 \\
(32.5 \%)\end{array}$ & $\begin{array}{l}527 \\
(28.2 \%)\end{array}$ & 0.22 & $\begin{array}{l}85 \\
(35.0 \%)\end{array}$ & $\begin{array}{l}1,134 \\
(31.1 \%)\end{array}$ & 0.051 & $\begin{array}{l}140 \\
(35.3 \%)\end{array}$ & $\begin{array}{l}1,644 \\
(29.1 \%)\end{array}$ & 0.031 & $\begin{array}{l}106 \\
(31.2 \%)\end{array}$ & $\begin{array}{l}1,462 \\
(25.2 \%)\end{array}$ & 0.0 \\
\hline \multicolumn{13}{|l|}{ Nerve invasion } \\
\hline No & $\begin{array}{l}110 \\
(66.3 \%)\end{array}$ & $\begin{array}{l}1,330 \\
(71.2 \%)\end{array}$ & & $\begin{array}{l}153 \\
(63.0 \%)\end{array}$ & $\begin{array}{l}2,427 \\
(66.5 \%)\end{array}$ & & $\begin{array}{l}186 \\
(46.9 \%)\end{array}$ & $\begin{array}{l}3,094 \\
(54.7 \%)\end{array}$ & & $\begin{array}{l}117 \\
(34.4 \%)\end{array}$ & $\begin{array}{l}2,120 \\
(36.5 \%)\end{array}$ & \\
\hline Yes & $2(1.2 \%)$ & $\begin{array}{l}13 \\
(0.7 \%)\end{array}$ & & $5(2.1 \%)$ & $\begin{array}{l}100 \\
(2.7 \%)\end{array}$ & & $\begin{array}{l}69 \\
(17.4 \%)\end{array}$ & $\begin{array}{l}917 \\
(16.2 \%)\end{array}$ & & $\begin{array}{l}122 \\
(35.9 \%)\end{array}$ & $\begin{array}{l}2,321 \\
(40.0 \%)\end{array}$ & \\
\hline Unknown & $\begin{array}{l}54 \\
(32.5 \%)\end{array}$ & $\begin{array}{l}526 \\
(28.1 \%)\end{array}$ & 0.35 & $\begin{array}{l}85 \\
(35.0 \%)\end{array}$ & $\begin{array}{l}1,125 \\
(30.8 \%)\end{array}$ & 0.35 & $\begin{array}{l}142 \\
(35.8 \%)\end{array}$ & $\begin{array}{l}1,646 \\
(29.1 \%)\end{array}$ & 0.0064 & $\begin{array}{l}101 \\
(29.7 \%)\end{array}$ & $\begin{array}{l}1,369 \\
(23.6 \%)\end{array}$ & 0.0 \\
\hline \multicolumn{13}{|l|}{ Therapeutic regimen } \\
\hline Surgery only & $3(1.8 \%)$ & $\begin{array}{l}99 \\
(5.3 \%)\end{array}$ & & $\begin{array}{l}12 \\
(4.9 \%)\end{array}$ & $\begin{array}{l}149 \\
(4.1 \%)\end{array}$ & & $\begin{array}{l}19 \\
(4.8 \%)\end{array}$ & $\begin{array}{l}347 \\
(6.1 \%)\end{array}$ & & $\begin{array}{l}55 \\
(16.2 \%)\end{array}$ & $\begin{array}{l}871 \\
(15.0 \%)\end{array}$ & \\
\hline $\begin{array}{l}\text { Multimodality } \\
\text { treatment }\end{array}$ & $\begin{array}{l}54 \\
(32.5 \%)\end{array}$ & $\begin{array}{l}452 \\
(24.2 \%)\end{array}$ & & $\begin{array}{l}72 \\
(29.6 \%)\end{array}$ & $\begin{array}{l}908 \\
(24.9 \%)\end{array}$ & & $\begin{array}{l}166 \\
(41.8 \%)\end{array}$ & $\begin{array}{l}1,800 \\
(31.8 \%)\end{array}$ & & $\begin{array}{l}149 \\
(43.8 \%)\end{array}$ & $\begin{array}{l}2,052 \\
(35.3 \%)\end{array}$ & \\
\hline Unknown & $\begin{array}{l}109 \\
(65.7 \%)\end{array}$ & $\begin{array}{l}1,318 \\
(70.5 \%)\end{array}$ & 0.014 & $\begin{array}{l}159 \\
(65.4 \%)\end{array}$ & $\begin{array}{l}2,595 \\
(71.1 \%)\end{array}$ & 0.18 & $\begin{array}{l}212 \\
(53.4 \%)\end{array}$ & $\begin{array}{l}3,510 \\
(62.1 \%)\end{array}$ & 0.0002 & $\begin{array}{l}136 \\
(40.0 \%)\end{array}$ & $\begin{array}{l}2,887 \\
(49.7 \%)\end{array}$ & 0.0 \\
\hline \multicolumn{13}{|l|}{ Surgical margin } \\
\hline Negative & $\begin{array}{l}120 \\
(72.3 \%)\end{array}$ & $\begin{array}{l}1,355 \\
(72.5 \%)\end{array}$ & & $\begin{array}{l}173 \\
(71.2 \%)\end{array}$ & $\begin{array}{l}2,650 \\
(72.6 \%)\end{array}$ & & $\begin{array}{l}265 \\
(66.8 \%)\end{array}$ & $\begin{array}{l}4,110 \\
(72.7 \%)\end{array}$ & & $\begin{array}{l}251 \\
(73.8 \%)\end{array}$ & $\begin{array}{l}4,417 \\
(76.0 \%)\end{array}$ & \\
\hline Positive & $\begin{array}{l}11 \\
(6.6 \%)\end{array}$ & $\begin{array}{l}110 \\
(5.9 \%)\end{array}$ & 0.92 & $4(1.7 \%)$ & $\begin{array}{l}103 \\
(2.8 \%)\end{array}$ & 0.41 & $8(2.0 \%)$ & $\begin{array}{l}106 \\
(1.9 \%)\end{array}$ & 0.037 & $7(2.1 \%)$ & $\begin{array}{l}92 \\
(1.6 \%)\end{array}$ & 0.5 \\
\hline
\end{tabular}

As for tumor, younger patients were more common in distal location (77.2\% versus $53.5 \%, \mathrm{P}<0.0001)$, Borrmann IV (8.4\% versus $5.8 \%$, $\mathrm{P}<0.05)$, poor differentiation $(53.7 \%$ versus $33.8 \%, P<0.0001)$, and pTNM stage IV $(19.5 \%$ versus $11.8 \%, P<0.001)$. The percentage of negative surgical margin in the patients aged $\geq 40$ was higher than that in younger patients, but there were no significant statistical differences in all four periods (all $P \geq 0.05$ ). Among patients in period 3 and period 4, GC in the younger group was more likely to present with diffuse classification $(P<0.0001)$, and also had more lymphatic invasion and vascular invasion ( $P=0.043, P=0.0008, P=0.031, P=0.0006$, resp.). In period 1 , period 3 and period 4 , younger patients with $G C$ also demonstrated a better acceptance of multimodality treatment ( $32.5 \%$ versus $24.2 \%, P=0.014 ; 41.8 \%$ versus $31.8 \%, P<0.001 ; 43.8 \%$ versus $35.3 \%, P=0.0016$ ).

Trends of clinicopathological features of younger patients with GC over the past 20 years were investigated. The median proportion of patients in the younger group over time was 6.2\% (range 5.5-8.2\%). No trend for the proportion of young patients from period 2 to period 4 was noted on linear regression $(P=0.053)$. The proportion of HER2 score of $0(-)$ and $1(+)$ had increased from period 3 to period $4(22.7-35.3 \%, 24.2-31.5 \%$, resp.), while the score of 2(++) and 3(+++) showed downward trends $(6.8-5.6 \%, 3.0-2.1 \%$, resp.). As shown in Fig. 1, the percentages of pTNM tumor stage I and pTNM stage II in the younger group had increased gradually with time (from $10.2 \%$ in period 1 to $22.7 \%$ in period 4 , from $6.0 \%$ in period 1 to $10.0 \%$ in period 4 , resp.), whereas GC in pTNM stage III and PTNM stage IV showed a decrease (from $51.2 \%$ in period 1 to $37.7 \%$ in period 4 , from $24.1 \%$ in period 1 to $15.9 \%$ in period 4 , resp.). In the therapeutic regimen, both the percentages of younger patients undergoing surgery and the patients after multimodality treatment had increased from period 1 to period 4 (1.8$16.2 \%, 32.5-42.8 \%$, resp.). As for surgical margin, a significant decrease was observed in positive margin for the younger group during 20 years. (from $6.6 \%$ in period 1 to $2.1 \%$ in period 4$)$.

\section{OS and PFS for younger patients with GC}

Changing trends of OS and PFS between younger and older groups were shown in Table 2 and Fig. 2. In general, a noticeable survival increment of GC was demonstrated in Cancer Center during 20 years. The 5-year OS in younger and older group has increased from $44.4 \%(95 \% \mathrm{Cl}$ : $28.2 \%-60.7 \%)$ to $86.0 \%$ ( $95 \% \mathrm{Cl}$ : $81.7 \%-90.4 \%$ ) and from $40.4 \%$ (95\% Cl: $36.0 \%-44.8 \%$ ) to $86.3 \%$ ( $95 \% \mathrm{Cl}: 85.2 \%-87.4 \%)$, respectively. After stratification by pTNM stage, the increased survival of younger cases was pronounced in stages III and IV (31.3\% in period 1 to $83.6 \%$ in period $4,0 \%$ in period 1 to $55.5 \%$ in period 4 , resp.). Moreover, the 5 -year PFS of younger patients has increased from $17.1 \%(95 \% \mathrm{Cl}: 8.3 \%-26.0 \%)$ in period 1 to $65.5 \%(95 \% \mathrm{Cl}$ : $58.4 \%-72.6 \%)$ in period 4 , with an obvious dominance in pTNM stage III (8.1-62\%). 
Table 2

The 5-year overall and progression-free survival rates by cancer stages (bidirectional cohort 1998-2018).

\begin{tabular}{|c|c|c|c|c|c|}
\hline Survival analysis & $\begin{array}{l}\text { Total, \% } \\
\text { (95\% Cl) }\end{array}$ & $\begin{array}{l}\text { PTNM stage I, \% } \\
(95 \% \mathrm{Cl})\end{array}$ & $\begin{array}{l}\text { PTNM stage II, \% } \\
(95 \% \mathrm{Cl})\end{array}$ & $\begin{array}{l}\text { pTNM stage III, \% } \\
(95 \% \mathrm{Cl})\end{array}$ & $\begin{array}{l}\text { PTNM stage IV, \% } \\
(95 \% \mathrm{Cl})\end{array}$ \\
\hline \multicolumn{6}{|l|}{ Total } \\
\hline \multicolumn{6}{|l|}{ Overall survival } \\
\hline Total $(n=13,533)$ & $68.1(67.2-68.9)$ & $96.6(95.8-97.3)$ & $85.1(83.3-86.8)$ & $61.8(60.4-63.2)$ & $19.0(16.3-21.8)$ \\
\hline Younger $(n=837)$ & $61.8(58.2-65.4)$ & $99.5(98.5-100.0)$ & $80.6(71.6-89.5)$ & $55.1(49.0-61.2)$ & $16.2(8.4-24.0)$ \\
\hline Older $(n=12,696)$ & $68.5(67.6-69.4)$ & $96.3(95.5-97.1)$ & $85.3(83.5-87.0)$ & $62.3(60.8-63.7)$ & $19.4(16.5-22.4)$ \\
\hline \multicolumn{6}{|l|}{ Progression-free survival } \\
\hline Total $(n=12,728)$ & $53.4(52.4-54.3)$ & $90.4(89.1-91.7)$ & $75.4(73.2-77.5)$ & $46.8(45.4-48.2)$ & $8.5(6.6-10.4)$ \\
\hline Younger $(n=856)$ & $46.0(42.4-49.6)$ & $95.6(92.4-98.8)$ & $63.1(52.2-74.1)$ & $36.3(30.7-42.0)$ & $8.7(3.6-13.8)$ \\
\hline Older $(n=11,872)$ & $53.9(53.0-54.9)$ & $89.9(88.6-91.3)$ & $76.0(73.9-78.2)$ & $47.5(46.0-49.0)$ & $8.4(6.3-10.4)$ \\
\hline \multicolumn{6}{|l|}{ Period 1} \\
\hline \multicolumn{6}{|l|}{ Overall survival } \\
\hline Total $(n=511)$ & $40.7(36.4-45.0)$ & $94.0(88.9-99.1)$ & $78.3(61.4-95.1)$ & $32.4(26.9-37.8)$ & $4.8(0.0-10.2)$ \\
\hline Younger $(n=36)$ & $44.4(28.2-60.7)$ & $100.0(100.0-100.0)$ & $100.0(100.0-100.0)$ & $31.3(8.5-54.0)$ & $0.0(0.0-0.0)$ \\
\hline Older $(n=475)$ & $40.4(36.0-44.8)$ & $93.3(87.7-99.0)$ & $75.0(56.0-94.0)$ & $32.5(26.8-38.1)$ & $5.4(0.0-11.3)$ \\
\hline \multicolumn{6}{|l|}{ Progression-free survival } \\
\hline Total $(n=794)$ & $26.5(23.4-29.5)$ & $79.8(71.9-87.7)$ & $42.4(25.6-59.3)$ & $21.7(17.9-25.4)$ & $2.2(0.0-5.1)$ \\
\hline Younger $(n=70)$ & $17.1(8.3-26.0)$ & $88.9(68.3-100.0)$ & $16.7(0.0-46.5)$ & $8.1(0.0-16.9)$ & $0.0(0.0-0.0)$ \\
\hline Older $(n=724)$ & $27.4(24.1-30.6)$ & $78.9(70.5-87.3)$ & $48.2(29.3-67.0)$ & $22.8(18.8-26.8)$ & $2.4(0.0-5.7)$ \\
\hline \multicolumn{6}{|l|}{ Period 2} \\
\hline \multicolumn{6}{|l|}{ Overall survival } \\
\hline Total $(n=2,801)$ & $60.7(58.9-62.5)$ & $93.9(91.7-96.2)$ & $77.2(72.0-82.4)$ & $57.5(54.9-60.1)$ & $12.2(8.0-16.3)$ \\
\hline Younger $(n=178)$ & $56.7(49.5-64.0)$ & $100.0(100.0-100.0)$ & $66.7(46.5-86.8)$ & $45.7(34.0-5.74)$ & $16.7(3.3-30.0)$ \\
\hline Older $(n=2,623)$ & $60.9(59.1-62.8)$ & $93.4(90.9-95.8)$ & $78.2(72.8-83.5)$ & $58.2(55.5-60.8)$ & $11.5(7.2-15.9)$ \\
\hline \multicolumn{6}{|l|}{ Progression-free survival } \\
\hline Total $(n=2,561)$ & $47.3(45.4-49.2)$ & $87.8(84.5-91.0)$ & $67.9(62.0-73.7)$ & $45.4(42.7-48.0)$ & $9.8(6.1-13.4)$ \\
\hline Younger $(n=176)$ & $46.0(38.7-53.4)$ & $94.3(86.6-100.0)$ & $52.2(31.7-72.6)$ & $37.3(26.4-48.3)$ & $16.1(3.2-29.1)$ \\
\hline Older $(n=2,385)$ & $47.4(45.4-49.4)$ & $87.1(83.6-90.6)$ & $69.5(63.5-75.5)$ & $45.9(43.1-48.6)$ & $8.9(5.2-12.6)$ \\
\hline \multicolumn{6}{|l|}{ Period 3} \\
\hline \multicolumn{6}{|l|}{ Overall survival } \\
\hline Total $(n=5,211)$ & $63.8(62.5-65.1)$ & $96.6(95.5-97.7)$ & $83.3(80.8-85.7)$ & $58.3(56.2-60.5)$ & $9.4(6.9-11.9)$ \\
\hline Younger $(n=331)$ & $52.9(47.5-58.2)$ & $98.6(95.7-100.0)$ & $80.0(66.8-93.2)$ & $49.2(40.2-58.1)$ & $6.7(0.4-13.0)$ \\
\hline Older $(n=4,880)$ & $64.6(63.2-65.9)$ & $96.4(95.3-97.6)$ & $83.4(80.9-85.8)$ & $58.9(56.7-61.1)$ & $9.8(7.0-12.5)$ \\
\hline \multicolumn{6}{|l|}{ Progression-free survival } \\
\hline Total $(n=4,635)$ & $50.1(48.6-51.5)$ & $91.3(89.4-93.2)$ & $74.4(71.4-77.5)$ & $43.7(41.4-45.9)$ & $4.3(2.6-6.0)$ \\
\hline Younger $(n=318)$ & $39.6(34.2-45.0)$ & $96.4(91.6-100.0)$ & $71.9(56.3-87.5)$ & 31.7 (23.5-39.9) & $5.9(0.3-11.5)$ \\
\hline Older $(n=4,317)$ & $50.9(49.4-52.3)$ & $90.9(88.9-92.9)$ & $74.5(71.5-77.6)$ & $44.5(42.2-46.9)$ & $4.1(2.4-5.9)$ \\
\hline \multicolumn{6}{|l|}{ Period 4} \\
\hline Overall survival & & & & & \\
\hline
\end{tabular}




\begin{tabular}{|clllll|}
\hline Total $(\mathrm{n}=5,010)$ & $86.3(85.2-87.3)$ & $98.7(98.0-99.4)$ & $94.1(92.3-95.9)$ & $83.5(81.5-85.5)$ & $53.0(47.0-59.0)$ \\
\hline Younger $(\mathrm{n}=292)$ & $86.0(81.7-90.4)$ & $100.0(100.0-100.0)$ & $96.9(90.8-100.0)$ & $83.6(75.8-91.4)$ & $55.5(37.5-73.5)$ \\
\hline Older $(\mathrm{n}=4,718)$ & $86.3(85.2-87.4)$ & $98.6(97.9-99.3)$ & $94.0(92.1-95.8)$ & $83.5(81.4-85.6)$ & $52.8(46.5-59.1)$ \\
\hline Progression-free survival & & & & & \\
\hline Total $(\mathrm{n}=4,738)$ & $69.8(67.9-71.7)$ & $92.2(89.4-94.9)$ & $84.8(81.9-87.7)$ & $66.5(63.3-69.6)$ & $6.8(0.3-13.4)$ \\
\hline Younger $(\mathrm{n}=292)$ & $65.5(58.4-72.6)$ & $95.3(88.6-100.0)$ & $80.3(60.7-99.9)$ & $62.0(50.9-73.1)$ & $0.0(0.0-0.0)$ \\
\hline Older $(n=4,446)$ & $70.1(68.1-72.0)$ & $91.9(89.0-94.9)$ & $85.0(82.1-87.9)$ & $66.8(63.5-70.0)$ & $7.7(0.4-15.1)$ \\
\hline
\end{tabular}

Figure 3 revealed the Kaplan-Meier curves for OS between the younger and older groups (Fig. 3). The analysis showed younger patients had a worse survival outcome in stage III $(P=0.0095)$ but a better prognosis in stage I $(P=0.03)$. However, no significant difference was found in stage II and stage IV $(P=0.60, P=$ 0.37 , resp.).

\section{Prognostic Factors in Univariate and Multivariate Analyses}

To investigate the significant factors impacting survival outcomes in young patients with GC, univariate and multivariate analyses were performed. The results revealed significantly different survival of GC based on the following parameters (Table 3): the period of diagnosis, drinking consumption, overweight/obesity (BMI $\geq 23,<27.4$ ), Weight loss $\geq 10 \%$, H. pylori infection, distal tumor location, Borrmann IV, Linitis plastic, poor differentiation, pTNM stage II, III, and IV, Vascular invasion, and surgical margin (all $\mathrm{P}<0.05$ ). For the younger adults, prognosis factors only involved diagnosis period $4(\mathrm{P}<0.0001)$, distal tumor location $(P=0.0007)$, Linitis plastica $(P=0.024)$, pTNM stage III $(P<0.0001)$, pTNM stage IV $(P<0.0001)$, and surgical margin $(P=0.011)($ Supplementary Table 1). 
Table 3

Univariate and multivariate survival analysis of predictors associated with overall survival for gastric cancer (bidirectional cohort 1998-2018).

Prognostic Factors

Univariate analysis

Hazard ratio

$95 \% \mathrm{Cl}$

Period of diagnosis

Period 1 (1998-2003)

Period 2 (2004-2008)

Period 3 (2009-2013)

Period 4 (2014-2018)

Gender

Male

1.00

Female

1.06

$0.99-1.14$

0.091

\section{Smoking history}

0.84-0.96

$0.89-1.25$

0.0009

0.54

00

No

Yes

Unknown

Drinking history

No
Yes

1.00

0.90

1.06

1.00

0.88

1.06

1.17

1.00

0.80

0.75

1.51

Unknown

Weight loss

None

$<10 \%$

$\geq 10 \%$

Unknown

H. pylori infection

Negative

Positive

Unknown

Primary tumor location

Proximal

Distal

Overlapping lesions

Unknown

Lauren classification

Intestinal

Diffuse

Mixed

$\mathrm{BMI}\left(\mathrm{kg} / \mathrm{m}^{2}\right)$ at diagnosis

$\begin{array}{ll}18.5-22.9 & 1.00 \\ 23-27.4 & 0.80 \\ \geq 27.5 & 0.75 \\ \text { Unknown } & 1.51\end{array}$

0

1.00

1.38

2.14

1.82

1.00

1.64

3.09

1.00

0.87
Multivariate anlaysis

Hazard ratio $\quad 95 \% \mathrm{Cl}$

1.00

$<0.0001 \quad 0.55$

$<0.0001 \quad 0.60$

$<0.0001 \quad 0.24$

$0.20-0.28$

$<0.0001$

$<0.0001$

$<0.0001$

P Value

1.00

$\begin{array}{lll}0.82-0.94 & 0.0001 & 0.93 \\ 0.89-1.25 & 0.52 & 0.86\end{array}$

$0.87-1.00$

0.045

$0.70-1.07$

0.18

$1.04-1.32$

0.0080

1.05

$0.93-1.19$

0.42

80

0.74-0.85

$<0.0001$

1.00

$0.68-0.83$

$<0.0001$

0.93

0.86-1.00

0.036

$1.31-1.74$

$<0.0001$

0.94

$0.85-1.05$

0.25

1.14

$0.96-1.35$

0.14

$\begin{array}{lll} & & 1.00 \\ 1.28-1.48 & <0.0001 & 1.05 \\ 1.95-2.35 & <0.0001 & 1.22 \\ 1.67-1.99 & <0.0001 & 1.07\end{array}$

1.05

1.22

1.07

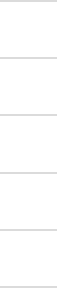

$+2$

$\begin{array}{ll}0.97-1.13 & 0.22 \\ 1.11-1.35 & <0.0001 \\ 0.97-1.19 & 0.16\end{array}$

\begin{tabular}{ll} 
& 1.00 \\
\hline$<0.0001$ & 1.29 \\
\hline 0.0001 & 1.54
\end{tabular}

$1.32-2.05$

$1.03-1.61$
$1.30-1.83$

0.024

$<0.0001$

$2.62-3.66$

0.81-0.92

1.00

1.36-1.78

$<0.0001$

0.93

$<0.0001 \quad 1.10$

$1.02-1.40$

0.026

0.65

1.00

$\begin{array}{lll}1.58-2.12 & <0.0001 & 1.17 \\ 1.14-1.61 & 0.0005 & 0.97\end{array}$

0.97

$0.99-1.38$

0.069

$0.81-1.15$

0.70

Page 10/19 


\begin{tabular}{|c|c|c|c|c|c|c|}
\hline \multirow[t]{2}{*}{ Prognostic Factors } & \multicolumn{3}{|c|}{ Univariate analysis } & \multicolumn{3}{|c|}{ Multivariate anlaysis } \\
\hline & Hazard ratio & $95 \% \mathrm{Cl}$ & P Value & Hazard ratio & $95 \% \mathrm{Cl}$ & P Value \\
\hline Unknown & 3.75 & $3.33-4.23$ & $<0.0001$ & 1.09 & $0.93-1.27$ & 0.30 \\
\hline \multicolumn{7}{|l|}{ Borrmann classification } \\
\hline Borrmann I & 1.00 & & & 1.00 & & \\
\hline Borrmann II & 0.94 & $0.83-1.07$ & 0.37 & 0.90 & $0.79-1.02$ & 0.089 \\
\hline Borrmann III & 1.17 & $1.03-1.33$ & 0.019 & 0.97 & $0.85-1.11$ & 0.68 \\
\hline Borrmann IV & 2.48 & $2.13-2.88$ & $<0.0001$ & 1.40 & $1.19-1.65$ & $<0.0001$ \\
\hline Mixed & 0.42 & $0.25-0.70$ & 0.0009 & 0.97 & $0.57-1.63$ & 0.90 \\
\hline Unknown & 2.50 & $2.21-2.84$ & $<0.0001$ & 1.06 & $0.93-1.21$ & 0.42 \\
\hline \multicolumn{7}{|l|}{ Linitis plastica } \\
\hline No & 1.00 & & & 1.00 & & \\
\hline Yes & 2.33 & $1.84-2.94$ & $<0.0001$ & 1.29 & $1.01-1.64$ & 0.044 \\
\hline Unknown & 1.51 & $1.33-1.71$ & $<0.0001$ & 0.85 & $0.72-0.99$ & 0.042 \\
\hline \multicolumn{7}{|l|}{ Differentiation } \\
\hline Well & 1.00 & & & 1.00 & & \\
\hline Moderate & 3.10 & $2.03-4.74$ & $<0.0001$ & 1.45 & $0.94-2.22$ & 0.090 \\
\hline Poor & 4.48 & $2.94-6.83$ & $<0.0001$ & 1.56 & $1.02-2.40$ & 0.041 \\
\hline Undifferentiated & 0.001 & 0.001-999.999 & 0.91 & 0.000 & 0.000-999.999 & 0.90 \\
\hline Unknown & 9.73 & $6.39-14.81$ & $<0.0001$ & 1.59 & $1.02-2.48$ & 0.042 \\
\hline \multicolumn{7}{|l|}{ HER2 score } \\
\hline $0(-)$ & 1.00 & & & 1.00 & & \\
\hline $1(+)$ & 0.84 & $0.74-0.96$ & 0.0082 & 0.96 & $0.85-1.09$ & 0.54 \\
\hline $2(++)$ & 0.71 & $0.59-0.84$ & 0.0001 & 0.90 & $0.75-1.08$ & 0.27 \\
\hline $3(+++)$ & 1.05 & $0.85-1.29$ & 0.68 & 1.08 & $0.87-1.33$ & 0.51 \\
\hline Unknown & 2.41 & $2.20-2.64$ & $<0.0001$ & 1.21 & $1.06-1.37$ & 0.0042 \\
\hline \multicolumn{7}{|l|}{ Pathologic T-stage } \\
\hline $\mathrm{T} 0+\mathrm{Tis}$ & 2.67 & $1.17-6.08$ & 0.020 & 1.38 & $0.19-10.14$ & 0.75 \\
\hline $\mathrm{T} 1$ & 1.00 & & & 1.00 & & \\
\hline $\mathrm{T} 2$ & 2.72 & $2.10-3.51$ & $<0.0001$ & 1.44 & $1.07-1.95$ & 0.017 \\
\hline T3 & 7.01 & $5.70-8.62$ & $<0.0001$ & 2.10 & $1.51-2.92$ & $<0.0001$ \\
\hline $\mathrm{T} 4$ & 11.29 & $9.24-13.80$ & $<0.0001$ & 2.55 & $1.82-3.57$ & $<0.0001$ \\
\hline TX & 19.07 & $15.58-23.33$ & $<0.0001$ & 2.50 & $1.72-3.62$ & $<0.0001$ \\
\hline \multicolumn{7}{|l|}{ Pathologic N-stage } \\
\hline No & 1.00 & & & 1.00 & & \\
\hline $\mathrm{N} 1$ & 2.28 & $1.98-2,63$ & $<0.0001$ & 1.27 & $1.09-1.49$ & 0.0020 \\
\hline N2 & 3.52 & $3.09-4.00$ & $<0.0001$ & 1.74 & $1.48-2.04$ & $<0.0001$ \\
\hline N3 & 6.85 & $6.11-7.67$ & $<0.0001$ & 2.82 & $2.41-3.29$ & $<0.0001$ \\
\hline NX & 8.72 & $7.79-9.75$ & $<0.0001$ & 1.74 & $1.35-2.26$ & $<0.0001$ \\
\hline \multicolumn{7}{|l|}{ Pathologic M-stage } \\
\hline MO & 1.00 & & & & & \\
\hline M1 & 5.76 & $5.34-6.22$ & $<0.0001$ & & & \\
\hline$M X$ & 1.98 & $1.78-2.20$ & $<0.0001$ & & & \\
\hline
\end{tabular}




\begin{tabular}{|c|c|c|c|c|c|c|}
\hline \multirow[t]{2}{*}{ Prognostic Factors } & \multicolumn{3}{|c|}{ Univariate analysis } & \multicolumn{3}{|c|}{ Multivariate anlaysis } \\
\hline & Hazard ratio & $95 \% \mathrm{Cl}$ & P Value & Hazard ratio & $95 \% \mathrm{Cl}$ & P Value \\
\hline 0 & 2.53 & $1.04-6.19$ & 0.042 & 1.76 & $0.20-15.49$ & 0.61 \\
\hline I & 1.00 & & & 1.00 & & \\
\hline ॥ & 3.70 & $2.99-4.57$ & $<0.0001$ & 1.69 & $1.23-2.32$ & 0.0012 \\
\hline III & 10.42 & $8.66-12.54$ & $<0.0001$ & 1.85 & $1.29-2.66$ & 0.0009 \\
\hline IV & 39.04 & $32.23-47.29$ & $<0.0001$ & 5.04 & $3.42-7.42$ & $<0.0001$ \\
\hline Unknown & 13.19 & $10.88-15.99$ & $<0.0001$ & 1.80 & $1.21-2.68$ & 0.0036 \\
\hline \multicolumn{7}{|l|}{ Lymphatic invasion } \\
\hline No & 1.00 & & & & & \\
\hline Yes & 1.76 & $1.62-1.92$ & $<0.0001$ & & & \\
\hline Unknown & 3.73 & $3.47-4.00$ & $<0.0001$ & & & \\
\hline \multicolumn{7}{|l|}{ Vascular invasion } \\
\hline No & 1.00 & & & 1.00 & & \\
\hline Yes & 1.77 & $1.63-1.92$ & $<0.0001$ & 1.17 & $1.07-1.28$ & 0.0006 \\
\hline Unknown & 3.74 & $3.48-4.01$ & $<0.0001$ & 1.17 & $1.00-1.38$ & 0.054 \\
\hline \multicolumn{7}{|l|}{ Nerve invasion } \\
\hline No & 1.00 & & & & & \\
\hline Yes & 1.06 & $0.96-1.16$ & 0.23 & & & \\
\hline Unknown & 3.15 & $2.95-3.37$ & $<0.0001$ & & & \\
\hline \multicolumn{7}{|l|}{ Therapeutic regimen } \\
\hline Surgery only & 1.00 & & & 1.00 & & \\
\hline Multimodality treatment & 3.73 & $3.04-4.58$ & $<0.0001$ & 1.09 & $0.88-1.34$ & 0.44 \\
\hline Unknown & 5.67 & $4.64-6.93$ & $<0.0001$ & 1.28 & $1.04-1.57$ & 0.022 \\
\hline \multicolumn{7}{|l|}{ Surgical margin } \\
\hline Negative & 1.00 & & & 1.00 & & \\
\hline Positive & 2.47 & $2.08-2.94$ & $<0.0001$ & 1.24 & $1.04-1.48$ & 0.015 \\
\hline Unknown & 3.10 & $2.91-3.30$ & $<0.0001$ & 1.28 & $1.10-1.50$ & 0.0020 \\
\hline \multicolumn{7}{|l|}{ Age group } \\
\hline Younger group (< 40 years) & 1.00 & & & 1.00 & & \\
\hline Older group ( $\geq 40$ years) & 0.80 & $0.71-0.90$ & 0.0001 & 0.92 & $0.82-1.04$ & 0.20 \\
\hline
\end{tabular}

In the univariate analysis, older patients with $\mathrm{GC}$ had better survival outcomes than the younger $(\mathrm{HR}=0.80,95 \% \mathrm{Cl}: 0.71-0.90, \mathrm{P}=0.0001)$. After stratification by PTNM stage, compared to the older, younger group showed a better prognosis in stage I $(P=0.04)$ but worse in stage III ( $P<0.01)($ Supplementary Table 2$)$. However, the multivariate analysis demonstrated that younger age was not an independent factor for poor survival outcomes $(P=0.20)$. In subdivided $p T N M$ stages, a comparison presented that younger group was associated with worse survival outcomes in tumor stage III $(P=0.014)$, but no statistical significance in stages I, II, and IV ( $P=0.074,0.59$ and 0.76 , resp. $)$.

\section{Genetic alterations of younger patients with GC}

The genetic alterations of GC patients from the China National Cancer Center were shown in Fig. 4A and Supplementary Fig. 1. Of 29 eligible patients with cancer genomics data, 20 were older (68.9\%), and the rest of 9 were younger (31.1\%). Although no statistical disparity was found in the mutational profile, younger patients have a higher mutation frequency in $R H O A$ (33\% versus $15 \%), C D H 1(22 \%$ versus $5 \%), K M T 2 D(22 \%$ versus $10 \%), S M A D 4(11 \%$ versus $0 \%$ ), ARID1A(11\% versus 5\%), ATM (11\% versus 5\%), CTNNB1(11\% versus 5\%), and PIK3CA(11\% versus 5\%), while older patients had dominated in TP53(70\% versus $44 \%$ ), $L R P 1 B(30 \%$ versus $11 \%)$, FGFR2 (20\% versus $0 \%$ ), ARID2 (15\% versus $0 \%$ ), EP300 (15\% versus $0 \%$ ), GNAS( $15 \%$ versus $11 \%), I R S 2(15 \%$ versus $11 \%), B R C A 2(10 \%$ versus $0 \%)$, and $A P C$ ( $5 \%$ versus $0 \%)$. Moreover, a high-volume analysis of the relationship between genetic alterations and age groups was 
further performed via the TCGA and MSKCC databases (Fig. 4B and Supplementary Fig. 2). GC tissues from older patients had higher likelihoods of $L R P 1 B$ (30\% versus $9 \%, \mathrm{P}<0.05), K M T 2 D$ ( $13 \%$ versus $0 \%, \mathrm{P}<0.05), A P C(13 \%$ versus $2 \%, \mathrm{P}<0.05)$, GNAS mutations $(11 \%$ versus $1 \%, \mathrm{P}<0.05)$ than the younger. Similar to the China National Cancer Center, although no significant differences were found, younger patients had mutation predominance in $C D H 1$ ( $17 \%$ versus $9 \%$ ), RHOA (7\% versus 5\%), and CTNNB1 mutations (9\% versus 6\%). In addition, of the pooled 8 gastric adenocarcinoma datasets, high-TMB was closely associated with older age at diagnosis $(\mathrm{P}<0.0001)$ (Supplementary Fig. 3).

\section{Discussion}

Our study provided a comprehensive analysis of younger GC patients at clinical and molecular levels. A primary finding was the stable proportion and significant survival improvements of younger cases in the China National Cancer Center from 1988 to 2018 . Younger age was proven to be an independent prognostic factor for GC patients in pTNM stage III, while patients in stage I, stage II, and stage IV had similar survival among different age groups. Compare to the younger, a higher mutation frequency of $\angle R P 1 B, K M T 2 D, A P C$, and GNAS genes in older patients was demonstrated in the current sequencing analysis, which might partly explain the histopathological behaviors and prognosis between the young and old groups.

Consistent with the previous study [10-15, 23, 24], our findings revealed unique clinical features in younger patients, including female dominance, higher proportions of poor differentiation, and advanced tumor stage. Although the mechanisms for female predominance in younger patients were not clear, some studies indicated that hormonal factors might account for the sex difference [25, 26]. Apart from H. pylori infection [27] and some gene distinctions [28], the vague symptoms and delayed diagnosis were also considerable reasons for the advanced stage of younger patients [29, 30].

With an increase of $41.6 \%$ for the younger group and $45.9 \%$ for the older group, a significant 5 -year survival improvement of GC was revealed in our center, which was in line with the increasing trend of PTNM stages I and II, surgery, and multimodality treatment. In China, survival trends of GC could be attributed to an improvement of quality of clinical services, including the improved access to primary healthcare, greater availability of diagnostic facilities, as well as enhanced effectiveness of the treatment [4]. Until 2015, the screening and early detection programs including GC have expanded to 31 provinces [31]. Encouragingly, the detection rate of GC has increased steadily benefit from the large-scale application of upper gastrointestinal endoscopy [32]. In addition, the widespread decision-making by the multi-disciplinary team (MDT) [33] and the emergence of individually multimodal therapies [34] were also closely related to a better prognosis for GC.

To date, survival outcomes of younger patients were still controversial. Previous studies suggested younger patients had a worse survival [14, 15], while several analyses showed on difference $[12,13]$. Current data demonstrated a worse prognosis for younger patients in pTNM stage III, whereas other stages showed similar survival. Therefore, variations of prognosis between the younger and older group might be related to stage distributions. Compare to older patients, the higher proportion of multimodality treatment in younger reflected a better tolerance for GC, while more advanced stage and aggressive behaviors might be related to a worse survival in this subpopulation [14]. Besides, the reduced organ functions and rising risk of comorbidities also potentially effect GC prognosis that occurred with age [35]. In addition, although genomic profiles of younger patients were not yet fully ascertained, several studies demonstrated a strong association between certain gene mutations and the prognosis of GC among age groups $[16,17]$.

The present study observed the differences in the prevalence of several gene mutations between younger and older patients, indicating a specific mutational spectrum in younger patients with GC. Significantly, a higher prevalence of $\angle R P 1 B$ mutations were identified in old patients. $L R P 1 B$ encoded a unique lowdensity lipoprotein receptor (LDLR) that functioned in the binding and internalization of ligands [36]. Until now, the concrete functions of $L R P 1 B$ in carcinogenesis were controversial [37-40]. Considered as a tumor suppressor, several findings indicated the impaired $L R P 1 B$ expression could promote the proliferation, migration, and invasion of carcinoma cells [38, 39]. However, the latest update of the Network of Cancer Genes (NCG 6.0) listed $L R P 1 B$ as a potentially false-positive tumor suppressor [39]. Zhou et al. [40] also demonstrated $\angle R P 1 B$ was one of the recurrently mutated driver genes, which was strongly associated with the development of GC. Further analysis was required to investigate the pathobiological property of $L R P 1 B$ in GC.

We also observed that older patients had a higher likelihood of GNAS mutations than younger. GNAS was situated on chromosome 20q13.3 and encoded alpha subunit of the stimulatory G-protein [41]. It was suggested that mutations of GNAS caused the activation of Wnt and ERK1/2 MAPK pathways via autonomous synthesis of cyclic adenosine monophosphate (cAMP), thus promoting intestinal tumorigenesis [42]. After that, several studies demonstrated the frequent GNAS mutations in gastric adenocarcinoma [43,44]. Consistent to current study, Esser et al.[41] also indicated the expression of GNAS was more prevalent in well- and moderately-differentiated GC, which was potentially correlated with older patients. Although the underlying mechanism was currently unclear, GNAS mutation might potentially affect the progression of GC via the protein kinase A (PKA), MAPK, and Wnt signaling pathways [45].

As in the past reports $[46,47]$, our analysis showed older patients had a higher prevalence of $A P C$ mutation than younger. Long considered a canonical tumor suppressor, $A P C$ could inhibit the excessive proliferation of tumor cells via regulating Wnt signaling pathway [48]. Mutation of $A P C$ was common in $\mathrm{GC}$, particularly in well-differentiated adenocarcinoma [49,50]. Zhan et al. [51] proposed a strong synergy between $A P C$ alterations and MEK inhibitors in enhancing the signaling output of the Wnt cascades. Gerner et al. [52] also observed mutant $A P C$ was associated with the elevation of nitric oxide synthase 2 and the dysregulation of polyamine metabolism. These data suggested that $A P C$ mutation might contribute to the oncogenesis of $\mathrm{GC}$. In addition, a recent study also demonstrated a significant association target high expression of $A P C$ and adverse prognosis of GC patients [53]. The observed prognostic alteration could be the result of the disruption of Wnt cascades, metabolic dysregulation, or some other unknown mechanisms.

$K M T 2 D$ was among the most frequently mutated genes in some types of cancer including GC [54-57]. Through encoding histone methyltransferase, KMT2D was critically involved in the epigenetic and transcriptional regulation of certain tumor-associated genes [56-60]. Lv et al. [56] suggested that KMT2D potentially prompt prostate carcinogenesis and metastasis via activating LIFR and KLF4 gene. Later, Xiong et al. [57] confirmed that KMT2D might play a role in cell proliferation and apoptosis of GC via the down-regulation of PTEN and up-regulation of LIFR and KLF4. However, recent studies also found the inhibiting effects of KMT2D in medulloblastoma [58], lung tumors [59], and lymphoma [60], as KMT2D could activate some specific pro-apoptotic genes or

Page $13 / 19$ 
repress certain genes related to cell growth and survival pathways. The above results revealed that the KMT2D gene had different functions and biological effects depending on the type of cancer. Furthermore, several studies indicated that mutations of $K M T 2 D$ were substantially higher in older patients than in younger [61,62], and the age disparity was also demonstrated in our analysis. More experimental studies were necessary to elucidate the underlying functions of $K M T 2 D$ in GC among age groups.

Our study has several strengths. First, clinicopathological characteristics and survival trends of the younger patients from 1998 to 2018 were comprehensively described based on NCCGCDB, which might serve as a reference for a large population-based study in China. Second, our study, for the first time, suggested distinctive mutation disparities including $\angle R P 1 B, G N A S, A P C$, and KMT2D genes in younger and older patients, which might partly explain the progression and survival of GC among age groups in molecular levels. The major limitation of our study was the insufficient sequencing sample size, particularly for pTNM subtype analysis. As such, possibility might not be ruled out for some of the significant findings. Based on the TCGA and MSKCC, we further analyze a largervolume sequencing sample to compare genetic alterations among age groups. In addition, regional and racial disparity of younger patients from China and western counties might also exist, which could affect the clinicopathological and molecular property of GC potentially. Therefore, parts of findings from TCGA and MSKCC might not be strongly generalizable to Chinese patients. Finally, some variables including tumor markers, neoadjuvant therapy, and adjuvant therapy were also needed to compare between the younger and older patients.

\section{Conclusions}

Our study found a stable proportion of young cases and significant survival improvements of younger patients in the China National Cancer Center from 1998 to 2018. Multivariate analysis suggested that younger patients with pTNM stage III had a worse survival than older, while other tumor stages showed no difference. Distinctive genetic alterations were further identified in younger patients, thus improving precision and personalized medicine in this subpopulation. Further large-scale studies are warranted to investigate more molecular characteristics and related mechanisms for GC patients at younger age.

\section{Abbreviations}

GC: gastric cancer; NCCGCDB: China National Cancer Center Gastric Cancer Database; OS: overall survival; AJCC: the American Joint Committee on Cancer; PFS: progression-free survival; NGS: next-generation sequencing; SNVs: single nucleotide variants; INDELs: insertion-deletions; TMB: tumor mutational burden; HR: hazard ratio; LDLR: low-density lipoprotein receptor; CAMP: cyclic adenosine monophosphate; PKA: the protein kinase A

\section{Declarations}

\section{Ethics approval and consent to participate}

The authors are accountable for all aspects of the work in ensuring that questions related to the accuracy or integrity of any part of the work are appropriately investigated and resolved. This study was approved by the ethics committee of National Cancer Center/National Clinical Research Center for Cancer/Cancer Hospital, Chinese Academy of Medical Sciences and Peking Union Medical College (No. 17-156/1412).

\section{Consent for publication}

Informed consent was obtained from all participants for publication.

\section{Availability of data and materials}

All data generated during this study are included in this published article and its supplementary files.

\section{Competing interests}

No potential conflicts of interest were disclosed.

\section{Funding}

This work was supported in part by the National Key R\&D Program of China (No. 2017YFC0908300).

\section{Authors' contributions}

YC and DZ considered and designed the study. PN, HH and LZ performed experiments. TW, XZ and WW did data analyses. TW, XZ and WW collected tumor samples, tissue specimens and clinical data. PN, HH and LZ wrote the manuscript. YZ and CG contributed to the revision of manuscript. All authors read and approved the final manuscript.

\section{Acknowledgements}

We thank anonymous reviewers and editor for reading and commenting on the manuscript.

\section{References}


1. Ferlay J, Colombet M, Soerjomataram I, Mathers C, Parkin DM, Piñeros M, Znaor A, Bray F. Estimating the global cancer incidence and mortality in 2018: GLOBOCAN sources and methods. Int J Cancer. 2019;144(8):1941-1953.

2. Chen W, Zheng R, Baade PD, Zhang S, Zeng H, Bray F, Jemal A, Yu XQ, He J. Cancer statistics in China, 2015. CA Cancer J Clin. 2016;66(2):115-32.

3. Zeng H, Zheng R, Guo Y, Zhang S, Zou X, Wang N, Zhang L, Tang J, Chen J, Wei K, et al. Cancer survival in China, 2003-2005: a population-based study. Int J Cancer. 2015;136(8):1921-30.

4. Zeng H, Chen W, Zheng R, Zhang S, Ji JS, Zou X, Xia C, Sun K, Yang Z, Li H, et al. Changing cancer survival in China during 2003-15: a pooled analysis of 17 population-based cancer registries. Lancet Glob Health. 2018;6(5):e555-e567.

5. Camargo MC, Anderson WF, King JB, Correa P, Thomas CC, Rosenberg PS, Eheman CR, Rabkin CS. Divergent trends for gastric cancer incidence by anatomical subsite in US adults. Gut. 2011;60(12):1644-9.

6. Kulig J, Popiela T, Kolodziejczyk P, Sierzega M, Jedrys J, Szczepanik AM, Polish Gastric Cancer Study Group. Clinicopathological profile and long-term outcome in young adults with gastric cancer: multicenter evaluation of 214 patients. Langenbecks Arch Surg. 2008;393(1):37-43.

7. Yin J, Song JN, Bai ZG, Cai J, Zhang J, Zheng Z, Wu HW, Ye PP, Gao X, Zhang ZT. Gastric Cancer Mortality Trends in China (2006-2013) Reveal Increasing Mortality in Young Subjects. Anticancer Res. 2017;37(8):4671-4679.

8. Islami F, DeSantis CE, Jemal A. Incidence Trends of Esophageal and Gastric Cancer Subtypes by Race, Ethnicity, and Age in the United States, $1997-2014$. Clin Gastroenterol Hepatol. 2019;17(3):429-439.

9. Anderson WF, Camargo MC, Fraumeni JF Jr, Correa P, Rosenberg PS, Rabkin CS. Age-specific trends in incidence of noncardia gastric cancer in US adults. JAMA. 2010;303(17):1723-8.

10. Zhou F, Shi J, Fang C, Zou X, Huang Q. Gastric Carcinomas in Young (Younger than 40 Years) Chinese Patients: Clinicopathology, Family History, and Postresection Survival. Medicine (Baltimore). 2016;95(9):e2873.

11. Bautista MC, Jiang SF, Armstrong MA, Postlethwaite D, Li D. Impact of age on clinicopathological features and survival of patients with noncardia gastric adenocarcinoma. J Gastric Cancer. 2014;14(4):238-45.

12. Liu S, Feng F, Xu G, Liu Z, Tian Y, Guo M, Lian X, Cai L, Fan D, Zhang H. Clinicopathological features and prognosis of gastric cancer in young patients. BMC Cancer. 2016;16:478.

13. Tekesin K, Emin Gunes M, Tural D, Akar E, Zirtiloglu A, Karaca M, Selcukbiricik F, Bayrak S, Ozet A. Clinicopathological characteristics, prognosis and survival outcome of gastric cancer in young patients: A large cohort retrospective study. J BUON. 2019;24(2):672-678.

14. Tavares A, Gandra A, Viveiros F, Cidade C, Maciel J. Analysis of clinicopathologic characteristics and prognosis of gastric cancer in young and older patients. Pathol Oncol Res. 2013;19(1):111-7.

15. Saito H, Takaya S, Fukumoto Y, Osaki T, Tatebe S, Ikeguchi M. Clinicopathologic characteristics and prognosis of gastric cancer in young patients. Yonago Acta Med. 2012;55(3):57-61.

16. Wu CW, Chen MH, Huang KH, Chang SC, Fang WL, Lin CH, Chao Y, Lo SS, Li AF, Shyr YM. The clinicopathological characteristics and genetic alterations between younger and older gastric cancer patients with curative surgery. Aging (Albany NY). 2020;12(18):18137-18150.

17. Seo JY, Jin EH, Jo HJ, Yoon H, Shin CM, Park YS, Kim N, Jung HC, Lee DH. Clinicopathologic and molecular features associated with patient age in gastric cancer. World J Gastroenterol. 2015;21(22):6905-13.

18. Zhao L, Huang H, Zhao D, Wang C, Tian Y, Yuan X, Ma F, Ren H, Zhao Y, Aimaiti S, et al. Clinicopathological Characteristics and Prognosis of Proximal and Distal Gastric Cancer during 1997-2017 in China National Cancer Center. J Oncol. 2019;2019:9784039.

19. Zhao LL, Huang H, Wang Y, Wang TB, Zhou H, Ma FH, Ren H, Niu PH, Zhao DB, Chen YT. Lifestyle factors and long-term survival of gastric cancer patients: A large bidirectional cohort study from China. World J Gastroenterol. 2020;26(14):1613-1627.

20. Smith AW, Bellizzi KM, Keegan TH, Zebrack B, Chen VW, Neale AV, Hamilton AS, Shnorhavorian M, Lynch CF. Health-related quality of life of adolescent and young adult patients with cancer in the United States: the Adolescent and Young Adult Health Outcomes and Patient Experience study. J Clin Oncol. 2013;31(17):2136-45.

21. Adolescent, Group YAOPR, Closing the Gap: Research and Care Imperatives for Adolescents and Young Adults with Cancer (NIH Publication No. 06-6067), Department of Health and Human Services, National Institutes of Health, National Cancer Institute, and the LIVESTRONG Young Adult Alliance Bethesda, MD, 2006.

22. Gao J, Aksoy BA, Dogrusoz U, Dresdner G, Gross B, Sumer SO, Sun Y, Jacobsen A, Sinha R, Larsson E, et al. Integrative analysis of complex cancer genomics and clinical profiles using the cBioPortal. Sci Signal. 2013;6(269):pl1.

23. Song M, Kang D, Yang JJ, Choi JY, Sung H, Lee Y, Yoon HS, Choi Y, Kong SH, Lee HJ, et al. Age and sex interactions in gastric cancer incidence and mortality trends in Korea. Gastric Cancer. 2015;18(3):580-9.

24. Dhobi MA, Wani KA, Parray FQ, Wani RA, Wani ML, Peer GQ, Abdullah S, Wani IA, Wani MA, Shah MA, et al. Gastric cancer in young patients. Int J Surg Oncol. 2013;2013:981654.

25. Di Domenico M, Castoria G, Bilancio A, Migliaccio A, Auricchio F. Estradiol activation of human colon carcinoma-derived Caco-2 cell growth. Cancer Res. 1996;56(19):4516-21.

26. Wang Z, Butler LM, Wu AH, Koh WP, Jin A, Wang R, Yuan JM. Reproductive factors, hormone use and gastric cancer risk: The Singapore Chinese Health Study. Int J Cancer. 2016;138(12):2837-45.

27. Hirahashi M, Yao T, Matsumoto T, Nishiyama K, Oya M, lida M, Tsuneyoshi M. Intramucosal gastric adenocarcinoma of poorly differentiated type in the young is characterized by Helicobacter pylori infection and antral lymphoid hyperplasia. Mod Pathol. 2007;20(1):29-34. 
28. Gurzu S, Kadar Z, Sugimura H, Bara T, Bara T Jr, Halmaciu I, Jung I. Gastric cancer in young vs old Romanian patients: immunoprofile with emphasis on maspin and mena protein reactivity. APMIS. 2015;123(3):223-33.

29. Koea JB, Karpeh MS, Brennan MF. Gastric cancer in young patients: demographic, clinicopathological, and prognostic factors in 92 patients. Ann Surg Oncol. 2000;7(5):346-51.

30. Chung HW, Noh SH, Lim JB. Analysis of demographic characteristics in 3242 young age gastric cancer patients in Korea. World J Gastroenterol. 2010;16(2):256-63.

31. Zou XN. Epidemic trend, screening, and early detection and treatment of cancer in Chinese population. Cancer Biol Med. 2017;14(1):50-59.

32. Liu K, Yang K, Zhang W, Chen X, Chen X, Zhang B, Chen Z, Chen J, Zhao Y, Zhou Z, et al. Changes of Esophagogastric Junctional Adenocarcinoma and Gastroesophageal Reflux Disease Among Surgical Patients During 1988-2012: A Single-institution, High-volume Experience in China. Ann Surg. 2016;263(1):88-95.

33. Blazeby JM, Wilson L, Metcalfe C, Nicklin J, English R, Donovan JL. Analysis of clinical decision-making in multi-disciplinary cancer teams. Ann Oncol. 2006;17(3):457-60.

34. Giampieri R, Del Prete M, Cantini L, Baleani MG, Bittoni A, Maccaroni E, Berardi R. Optimal management of resected gastric cancer. Cancer Manag Res. 2018;10:1605-1618.

35. Joharatnam-Hogan N, Shiu KK, Khan K. Challenges in the treatment of gastric cancer in the older patient. Cancer Treat Rev. 2020;85:101980.

36. Go GW, Mani A. Low-density lipoprotein receptor (LDLR) family orchestrates cholesterol homeostasis. Yale J Biol Med. 2012;85(1):19-28.

37. Yasufuku I, Saigo C, Kito Y, Yoshida K, Takeuchi T. Prognostic significance of LDL receptor-related protein 1B in patients with gastric cancer. J Mol Histol. 2021;52(2):165-172.

38. Wang Z, Sun P, Gao C, Chen J, Li J, Chen Z, Xu M, Shao J, Zhang Y, Xie J. Down-regulation of LRP1B in colon cancer promoted the growth and migration of cancer cells. Exp Cell Res. 2017;357(1):1-8.

39. Repana D, Nulsen J, Dressler L, Bortolomeazzi M, Venkata SK, Tourna A, Yakovleva A, Palmieri T, Ciccarelli FD. The Network of Cancer Genes (NCG): a comprehensive catalogue of known and candidate cancer genes from cancer sequencing screens. Genome Biol. 2019;20(1):1.

40. Zhou J, Zhao W, Wu J, Lu J, Ding Y, Wu S, Wang H, Ding D, Mo F, Zhou Z, et al. Neoantigens Derived from Recurrently Mutated Genes as Potential Immunotherapy Targets for Gastric Cancer. Biomed Res Int. 2019;2019:8103142.

41. Esser D, Holze N, Haag J, Schreiber S, Krüger S, Warneke V. Interpreting whole genome and exome sequencing data of individual gastric cancer samples. BMC Genomics. 2017;18(1):517.

42. Wilson $\mathrm{CH}$, McIntyre RE, Arends MJ, Adams DJ. The activating mutation R201C in GNAS promotes intestinal tumourigenesis in Apc(Min/+) mice through activation of Wnt and ERK1/2 MAPK pathways. Oncogene. 2010;29(32):4567-75.

43. Nomura R, Saito T, Mitomi H, Hidaka Y, Lee SY, Watanabe S, Yao T. GNAS mutation as an alternative mechanism of activation of the Wnt/ $\beta$-catenin signaling pathway in gastric adenocarcinoma of the fundic gland type. Hum Pathol. 2014;45(12):2488-96.

44. Matsubara A, Sekine S, Kushima R, Ogawa R, Taniguchi $H$, Tsuda $H$, Kanai Y. Frequent GNAS and KRAS mutations in pyloric gland adenoma of the stomach and duodenum. J Pathol. 2013;229(4):579-87.

45. Alakus H, Babicky ML, Ghosh P, Yost S, Jepsen K, Dai Y, Arias A, Samuels ML, Mose ES, Schwab RB, et al. Genome-wide mutational landscape of mucinous carcinomatosis peritonei of appendiceal origin. Genome Med. 2014;6(5):43.

46. Wang F, Cheng H, Zhang X, Shan L, Bai B, Chen K, Lou F, Cao S, Wang H, Dai S. Comparative genomic signatures in young and old Chinese patients with colorectal cancer. Cancer Med. 2021;10(13):4375-4386.

47. Lieu CH, Golemis EA, Serebriiskii IG, Newberg J, Hemmerich A, Connelly C, Messersmith WA, Eng C, Eckhardt SG, Frampton G, et al. Comprehensive Genomic Landscapes in Early and Later Onset Colorectal Cancer. Clin Cancer Res. 2019;25(19):5852-5858.

48. Ghatak S, Chakraborty P, Sarkar SR, Chowdhury B, Bhaumik A, Kumar NS. Novel APC gene mutations associated with protein alteration in diffuse type gastric cancer. BMC Med Genet. 2017;18(1):61.

49. Nakatsuru S, Yanagisawa A, Ichii S, Tahara E, Kato Y, Nakamura Y, Horii A. Somatic mutation of the APC gene in gastric cancer: frequent mutations in very well differentiated adenocarcinoma and signet-ring cell carcinoma. Hum Mol Genet. 1992;1(8):559-63.

50. Sano T, Tsujino T, Yoshida K, Nakayama H, Haruma K, Ito H, Nakamura Y, Kajiyama G, Tahara E. Frequent loss of heterozygosity on chromosomes 1q, 5q, and 17p in human gastric carcinomas. Cancer Res. 1991;51(11):2926-31.

51. Zhan T, Ambrosi G, Wandmacher AM, Rauscher B, Betge J, Rindtorff N, Häussler RS, Hinsenkamp I, Bamberg L, Hessling B, et al. MEK inhibitors activate Wnt signalling and induce stem cell plasticity in colorectal cancer. Nat Commun. 2019;10(1):2197.

52. Gerner EW, Bruckheimer E, Cohen A. Cancer pharmacoprevention: Targeting polyamine metabolism to manage risk factors for colon cancer. J Biol Chem. 2018;293(48):18770-18778.

53. Du WB, Lin CH, Chen WB. High expression of APC is an unfavorable prognostic biomarker in T4 gastric cancer patients. World J Gastroenterol. 2019;25(31):4452-4467.

54. Hanna CW, Taudt A, Huang J, Gahurova L, Kranz A, Andrews S, Dean W, Stewart AF, Colomé-Tatché M, Kelsey G. MLL2 conveys transcription-independent H3K4 trimethylation in oocytes. Nat Struct Mol Biol. 2018;25(1):73-82.

55. Toska E, Osmanbeyoglu HU, Castel P, Chan C, Hendrickson RC, Elkabets M, Dickler MN, Scaltriti M, Leslie CS, Armstrong SA, et al. PI3K pathway regulates ER-dependent transcription in breast cancer through the epigenetic regulator KMT2D. Science. 2017;355(6331):1324-1330. 
56. Lv S, Ji L, Chen B, Liu S, Lei C, Liu X. Histone methyltransferase KMT2D sustains prostate carcinogenesis and metastasis via epigenetically activating LIFR and KLF4. Oncogene. 2018;37(10):1354-1368.

57. Xiong W, Deng Z, Tang Y, Deng Z, Li M. Downregulation of KMT2D suppresses proliferation and induces apoptosis of gastric cancer. Biochem Biophys Res Commun. 2018;504(1):129-136.

58. Dhar SS, Zhao D, Lin T, Gu B, Pal K, Wu SJ. MLL4 Is Required to Maintain Broad H3K4me3 Peaks and Super-Enhancers at Tumor Suppressor Genes. Mol Cell. 2018;70(5):825-841.e6.

59. Alam H, Tang M, Maitituoheti M, Dhar SS, Kumar M, Han CY, Ambati CR, Amin SB, Gu B, Chen TY, et al. KMT2D Deficiency Impairs Super-Enhancers to Confer a Glycolytic Vulnerability in Lung Cancer. Cancer Cell. 2020;37(4):599-617.e7.

60. Ortega-Molina A, Boss IW, Canela A, Pan H, Jiang Y, Zhao C, Jiang M, Hu D, Agirre X, Niesvizky I, et al. The histone lysine methyltransferase KMT2D sustains a gene expression program that represses B cell lymphoma development. Nat Med. 2015;21(10):1199-208.

61. Ramis-Zaldivar JE, Gonzalez-Farré B, Balagué O, Celis V, Nadeu F, Salmerón-Villalobos J, Andrés M, Martin-Guerrero I, Garrido-Pontnou M, Gaafar A, et al. Distinct molecular profile of IRF4-rearranged large B-cell lymphoma. Blood. 2020;135(4):274-286.

62. Ma D, Jiang YZ, Xiao Y, Xie MD, Zhao S, Jin X, Xu XE, Shao ZM. Integrated molecular profiling of young and elderly patients with triple-negative breast cancer indicates different biological bases and clinical management strategies. Cancer. 2020;126(14):3209-3218.

\section{Figures}

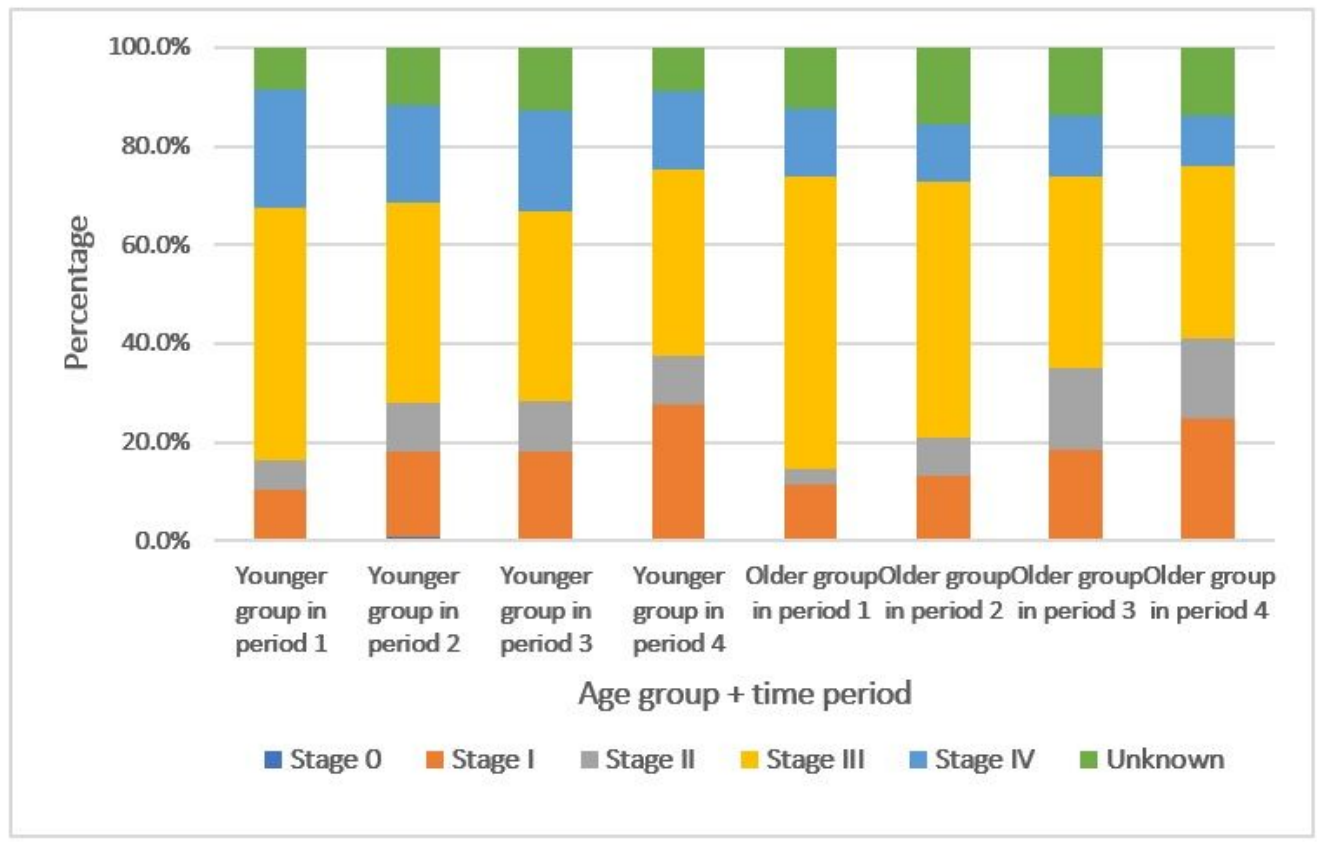

\section{Figure 1}

Changing trends of the proportion of younger and older groups by period and pTNM tumor stage.

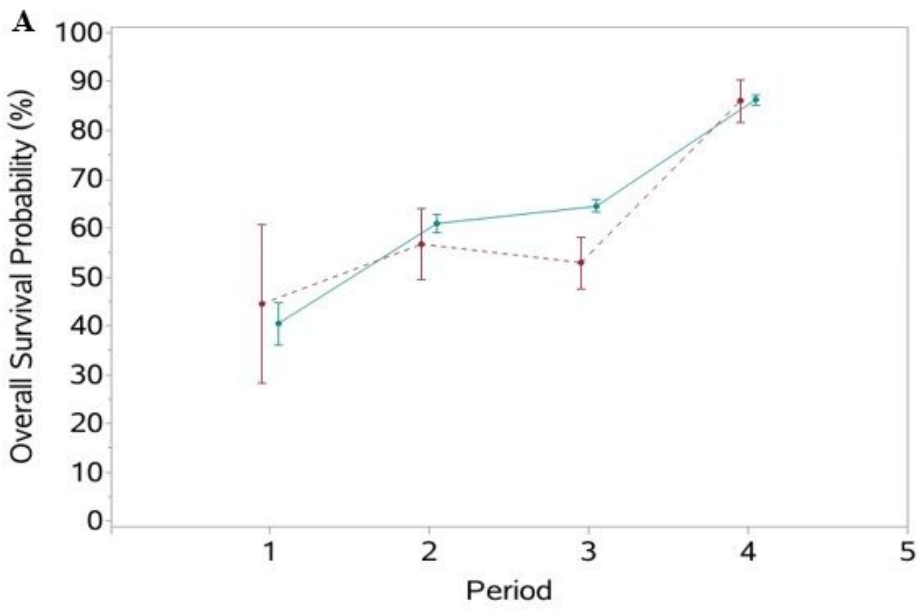

Age Group: — Older Group … Younger Group

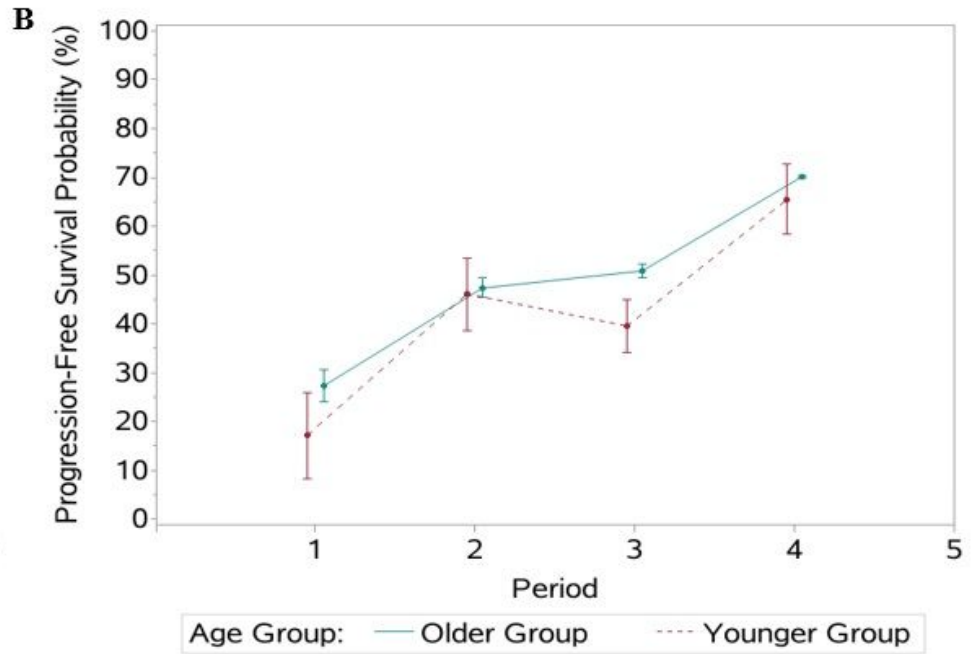


Figure 2

Survival trends between younger and older groups during 20 years. A: The 5-year overall survival. B: The 5-year progression-free survival
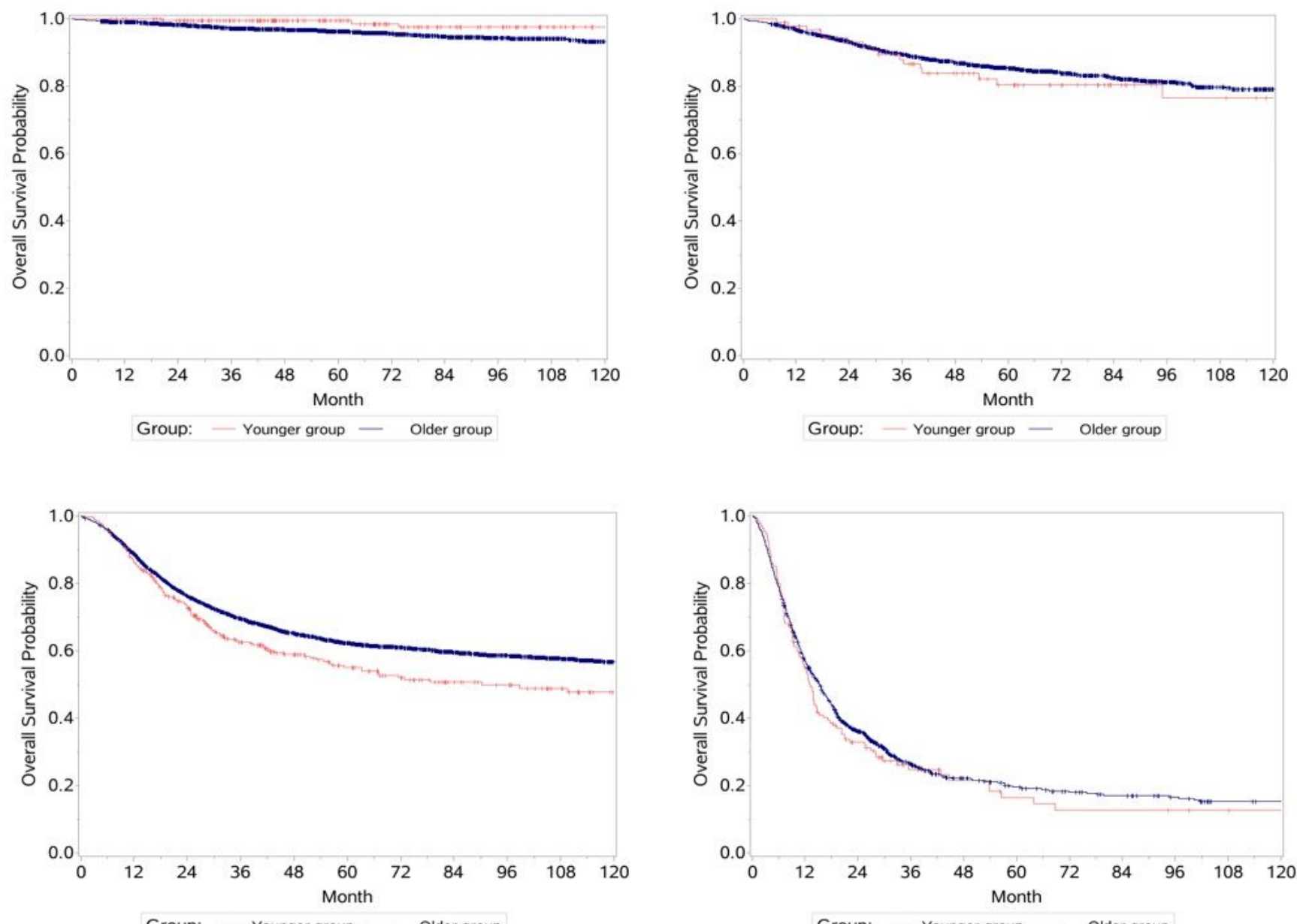

\section{Figure 3}

Kaplan-Meier survival curves of gastric cancer patients between YG and OG. A: Kaplan-Meier survival curves of patients in pTNM stage I. B: Kaplan-Meier survival curves of patients in pTNM stage II. C: Kaplan-Meier survival curves of patients in pTNM stage III. D: Kaplan-Meier survival curves of patients in pTNM stage IV. 

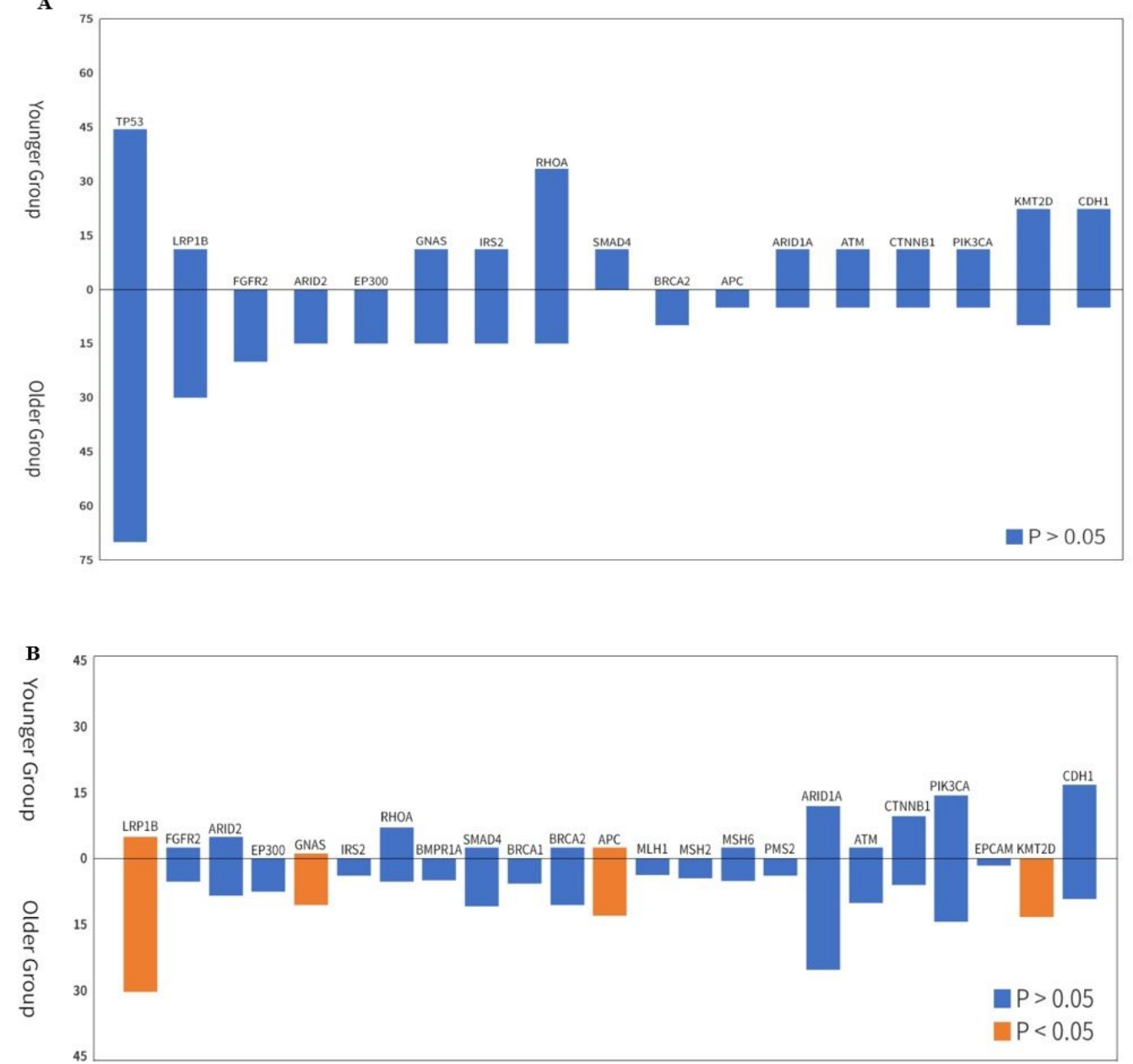

\section{Figure 4}

Comparison of mutation frequencies between younger and older groups. A: Genetic alterations from the China National Cancer Center. B: Genetic alterations from the TCGA and MSKCC databases.

\section{Supplementary Files}

This is a list of supplementary files associated with this preprint. Click to download.

- FigureS1.jpg

- Figures2.jpg

- FigureS3.png

- SupplementalDocument1.pdf

- SupplementalDocument2.pdf

- SupplementalTables.docx 